\title{
Kinetic Modeling and Design of Colloidal Lock and Key Assembly
}

\author{
Daniel J. Beltran-Villegas ${ }^{1}$ *, Laura Colón-Meléndez ${ }^{2}$, Michael J. Solomon ${ }^{1}$ and Ronald G. \\ Larson $^{1} *$ \\ ${ }^{1}$ Department of Chemical Engineering, University of Michigan, Ann Arbor, MI 48109 \\ ${ }^{2}$ Department of Physics, University of Michigan, Ann Arbor, MI 48109
}

\begin{abstract}
We investigate the kinetics of colloidal lock and key particle assembly by modeling transitions between free, non-specifically and specifically (dumbbells) bound pairs to enable the rapid formation of specific pairs. We expand on a model introduced in a previous publication (Colón-Meléndez, L., et. al., The Journal of Chemical Physics, 142 (2015) 174909) to account for the shape complementarity between the lock and the key particle. Specifically we develop a theory to predict free energy differences between specific and non-specific states based on the interaction potential between arbitrary surfaces and apply this to the interaction of a spherical key particle with the concave dimple surface. Our results show that a lock particle dimple slightly wider than the key particle radius results in optimal binding, but also show escape rates much smaller than those observed in experimental measurements described in the paper cited above. We assess the possible sources of error in experiments and in analysis, including spatial and temporal resolution of the confocal microscopy method used to measure kinetic coefficients, the polydispersity of the lock dimple size, and the sedimentation of the particles in a quasi-twodimensional layer. We find that the largest sources of variation are in the limited temporal resolution of the experiments, which we account for in our theory, and in the quasi-twodimensional nature of the experiment that leads to misidentification of non-specific pairs as specific ones. Accounting for these sources of variation results in very good quantitative agreement with experimental data.
\end{abstract}

Keywords: Kinetics of self-assembly; Anisotropic particle assembly; Lock-key colloids; Shape complementarity in lock-key systems

\section{Introduction}

Colloidal crystals are assemblies of micron-sized particles with emerging properties beyond those of the individual building blocks. Forming colloidal crystal structures different from close-packed arrays requires breaking the symmetry of isotropic interactions[1] by having either non-spherical shapes, such as ellipsoidal[2, 3] and polyhedral particles[4], or directional interactions, exemplified by patchy particles[5-9]. The types of structures that arise from different anisotropic building blocks have been studied extensively by means of equilibrium thermodynamics $[4,9,10]$; nonetheless the kinetics of structure formation have not been studied to the same extent. A common roadblock in the formation of complex lattices is the formation of

\footnotetext{
* To whom correspondence should be addressed. beltran@umich.edu, rlarson@umich.edu
} 
competing meta-stable structures[11, 12]. Hierarchical assembly, namely the assembly of intermediate structures, which are the building blocks of a more complex lattice, is a strategy that shows promise in the sense of guiding the lattice formation to desirable shapes. Lock and key colloids[13] offer a unique approach to the formation of a wide variety of intermediate structures, such as dumbbells, trimers, tetramers, and so forth, in a controlled fashion.

Colloidal locks are spherical particles that have a dimple where another particle, the key, fits. This system has been studied both experimentally[13-15] and theoretically[16, 17], specifically in a previous confocal microscopy study[14] different states and transitions between states were identified, where free (F) locks and keys can bind either non-specifically (NS, where the key is held close to the lock at a position different from the dimple) or specifically ( $\mathrm{S}$, where the key sits at the lock dimple), as summarized in Figure 1a. This binary system interacts by means of a depletion attraction[18] where the magnitude of the attractive potential is proportional to the overlap in volume excluded to non-adsorbing polymer present in solution, shown schematically in Fig 1b. The depletion interaction is much stronger for a specific bond than for a non-specific bond, driving the formation of dumbbells. Quantification of transition rates[14] was made by Smoluchowski collision rates[19] for F-S and F-NS events, and by a diffusion-migration model for NS-S and NS-F events, showing quantitative agreement with experimental data.

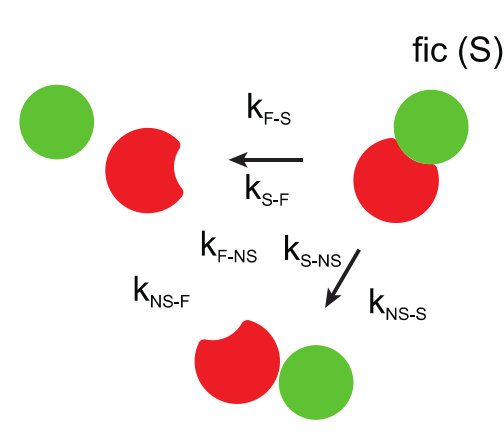

a
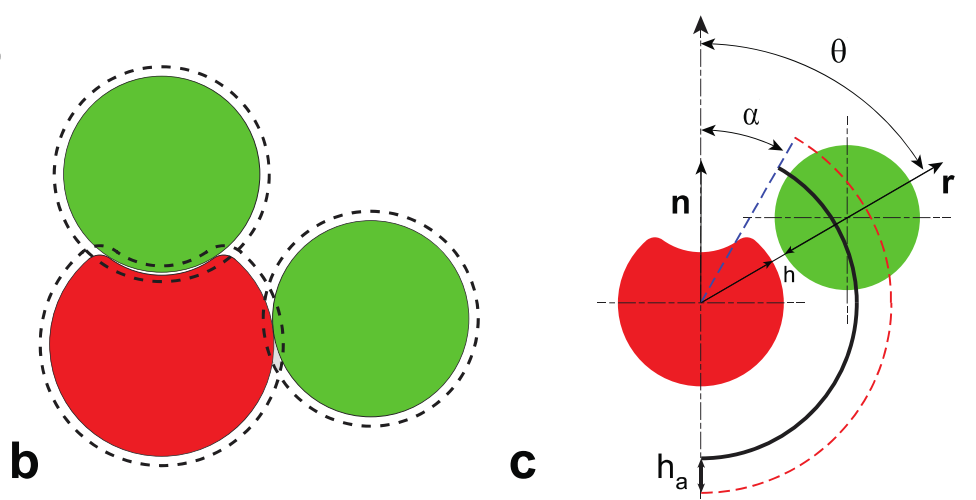

Fig 1. (a) schematic of Lock and Key particle pair configurations and transitions, adapted from a previous publication[14]. Free lock and key pairs (F) can form either a specific (S) pair, when the key sits in the dimple of the lock, or a non-specific (NS) pair, when the key is bound to the convex surface of the lock. (b) schematic of overlap excluded volume regions (shown as a shaded gray area) for lock and key particles interacting specifically or non-specifically. Depletion interaction is proportional to the overlap excluded volume region between a lock and a key; the interaction potential is strongest on a specific configuration, but a non-specific configuration can be held temporarily. (c) definition of important variables used in the modeling of kinetics, adapted from a previous publication[14]. Lock and key pair relative configuration can be expressed in terms of $h$, the surface-to-surface distance between particles, and $\theta$, the angle between the lock director and the center-to-center vector between particles. A specific pair is considered when $\theta<\alpha$, where a is the aperture of the dimple, and $h<h_{\mathrm{a}}$, where $h_{\mathrm{a}}$ is an escape distance. A non-specific pair is considered when $h<h_{\mathrm{a}}$ and $\theta>\alpha$. Other configurations, i.e. $h>h_{\mathrm{a}}$, correspond to free pairs.

In this work we re-visit the modeling of kinetic rates for lock and key colloid assembly. Specifically we expand the previously presented theory, first, to include kinetic estimations for $\mathrm{S}-\mathrm{NS} / \mathrm{F}$ transitions that include the estimation of interaction potentials between arbitrary shapes that allow the study of shape complementarity between the lock particle dimple and the key 
particle; second, to benchmark the diffusion-migration model for NS-S/F transitions with traditional Stokesian dynamic simulations; and third, to guide the design of colloidal lock and key assembly processes. After presenting the expanded theory we address experimental limitations and their effect in the analysis and interpretation of the transition kinetics, motivated by measured S-NS/F kinetic rate constants[14] higher than those predicted by our theory. Specifically we address issues related to (1) spatial resolution, which affects the perceived center-to-center distance between particles; (2) temporal resolution, which affects the measured rates for transition processes faster than the microscope frame acquisition rate; (3) distribution of lock dimple radius, which affects perceived population transition kinetics due to differences in shape complementarity; and (4) sedimentation of particles, which affects the perception of nonspecific pairs as false positive specific pairs, distorting the perceived kinetics. We include quantitative comparisons with experimental data to validate our findings.

The study of kinetics of lock and key colloid assembly, besides its technological implications to the robust design of novel technologies, sheds light on lock-and-key assembly processes at different scales, particularly those of enzyme-ligand and antibody-antigen interactions where shape complementarity plays an important role.

\section{Theory}

\section{Colloidal interactions}

\section{Sphere-sphere interaction potentials}

The total interaction potential energy between a lock and a key particle interacting nonspecifically (i.e. when the key particle is far from the lock particle dimple), $\phi^{\square}(r)$, is the sum of attractive depletion and repulsive DLVO potentials,

$$
\phi^{N S}(r)=\phi_{A O}^{N S}(r)+\phi_{D L V O}^{N S}(r)
$$

The depletion potential for two unequal spheres, $\phi^{\square} \mathrm{AO}(r)$, where $r$ is the center-to-center distance between particles, is calculated using the Asakura-Oosawa theory[18], and is given by,

$$
\phi_{A O}^{N S}(r)=-\Pi \Lambda V^{N S}(r)
$$

where $\Pi$ is the non-adsorbing depletant osmotic pressure and $\Delta V^{\mathrm{NS}}(r)$ is the overlap excluded volume region, shown in the shaded gray region in Fig $1 \mathrm{~b}$, given by,

$$
\Delta V^{N S}(r)=\frac{\pi\left(a_{L}^{\prime}+a_{K}^{\prime}-r\right)^{2}\left(r^{2}+2 r a_{K}^{\prime}-3 a_{K}^{\prime 2}+2 r a_{L}^{\prime}-3 a_{L}^{\prime 2}+6 a_{L}^{\prime} a_{K}^{\prime}\right)}{12 r},
$$

where $a^{\prime}{ }_{\mathrm{L}}=a_{\mathrm{L}+} a_{\mathrm{D}}, a_{\mathrm{L}}$ is the radius of the lock particle, $a_{\mathrm{D}}$ is the depletant radius, $a^{\prime}{ }_{\mathrm{K}}=a_{\mathrm{K}}+a_{\mathrm{D}}$, and $a_{\mathrm{K}}$ is the radius of the key particle.

Screened electrostatic repulsion between surfaces is calculated following the DLVO theory[19]. The DLVO potential for two unequal spheres, $\phi^{\mathrm{NS}}{ }_{\text {DLVO }}(r)$, is given by,

$$
\phi_{D L V O}^{N S}(r)=B_{P P} \exp \left[-\kappa\left(r-a_{L}-a_{K}\right)\right],
$$


where $B_{\mathrm{PP}}$ is a pre-exponential factor given by,

$$
B_{P P}=64 \pi \varepsilon\left(\frac{a_{L} a_{K}}{a_{L}+a_{K}}\right)\left(\frac{k T}{e}\right)^{2} \tanh \left(\frac{e \zeta_{L}}{4 k T}\right) \tanh \left(\frac{e \zeta_{K}}{4 k T}\right),
$$

$k$ is Boltzmann's constant, $T$ is the absolute temperature, $e$ is the elemental charge, $\varepsilon$ is the solvent dielectric permittivity, $\zeta_{\mathrm{L}}$ and $\zeta_{\mathrm{K}}$ are the zeta potentials of lock and key particles, respectively, $\kappa^{-1}$ is the Debye screening length, the inverse of which is given by,

$$
\kappa=\left(\frac{2 e^{2} N_{A} c_{e}}{\varepsilon k T}\right)^{1 / 2}
$$

$N_{\mathrm{A}}$ is Avogadro's number, and $c_{\mathrm{e}}$ is the 1:1 monovalent electrolyte molarity.

Additional interaction potentials can be included in our model, such as van der Waals forces[19] and adsorbed polymer steric interactions[20], nonetheless, the quantitative comparisons are made with an experimental system where van der Waals forces are effectively neutralized by adsorbed polymer steric interactions[14].

\section{General shape interaction potentials}

Past studies on depletion interactions have shown that depletion potentials can be greatly reduced[21], causing an effective "depletion barrier", in the proximity of an edge, as the overlap in excluded volume regions decreases as a particle interacting with a surface approaches a step in that surface. This "depletion barrier" effect can have an important effect in the vicinity of the lock dimple. Additionally, to determine the stability of the specific state, we need to evaluate the magnitude of the potential energy of interaction between lock and key at relative positions where the sphere-sphere interaction potentials are unrealistic. Previous studies[13] have used approximations to the potential energy of interaction in specific configurations, but these approximations rely on adjustable parameters for stability estimations. In this section we describe how general shape interaction potentials are calculated.

The depletion potential between surfaces of arbitrary shape takes a similar form to Eq (2), with the caveat that radial symmetry is broken. In the particular case of an axi-symmetrical lock interacting with a key, the general shape depletion potential, $\phi_{\mathrm{AO}}$, is given by

$$
\phi_{A O}(r, \theta)=-\Pi \Lambda V(r, \theta)
$$

where the overlap excluded volume region, $\Delta V(r, \theta)$, is estimated numerically for a given lock and key pair configuration. This numerical estimation is explained in the Methods section.

The screened electrostatic repulsion between arbitrary shapes has been the subject of different studies. Different approximations to general shapes include curvature-based Derjaguin approximations[22], valid for shapes where the radius of curvature of the intervening surfaces is much larger than the Debye screening length. In the particular case of lock and key particles, as shown in Fig 1b, a simple curvature-based Derjaguin approximation can be unsuitable in the case where the two intervening surfaces have multiple approach points (i.e. a non-monotonic curvature, such as a lock particle), or where the radii of curvature of complementary surfaces are very similar. Given that neither of the two surfaces is a flat plate, the approximation of a general 
surface interacting with a flat plate has to be changed to include this effect.

A general solution to the screened electrostatic repulsion interaction potential between arbitrary surfaces, $\phi_{\mathrm{DLVO}}$, can be expressed as,

$$
\phi_{D L V O}(r, \theta)=\int_{\mathbb{Q}_{L} \mathbb{S}_{K}} d A_{L} d A_{K} \cos \left(\psi_{L}\right) \cos \left(\psi_{K}\right) e_{D L V O}(l)
$$

where the function $e_{\mathrm{DLVO}}(l)$ is the interaction potential between two surface elements, $d A_{\mathrm{L}}$ and $d A_{\mathrm{K}}$, per unit area of surface "L" and surface " $\mathrm{K}$ ", and $l$ is the distance between area elements $d A_{\mathrm{L}}$ and $d A_{\mathrm{K}}$, shown in Fig 2a. Equation (8) can be related to the well-known solution for interaction between infinite plates, $E_{\mathrm{DLVO}}(H)[19,22]$, as

$$
\phi_{D L V O, W}(H)=\int_{\mathbb{S}_{L}} d A_{L} E_{D L V O}(H),
$$

where $H$ is the distance between the plates. The function $E_{\mathrm{DLVO}}(H)$ refers to the interaction potential between an infinite plate and a unit area of a parallel surface, shown in Fig $2 b$.

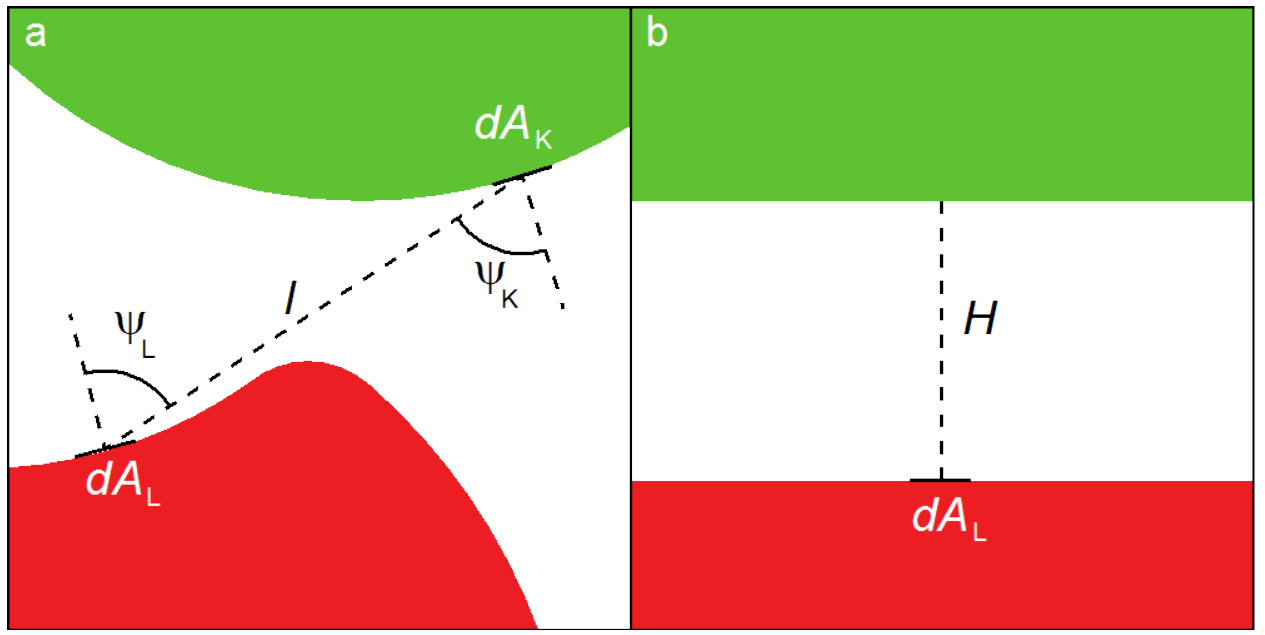

Fig 2. Schematic for DLVO potentials between surfaces. (a) two arbitrary surfaces "L" and "K". Schematic shows surface elements $d A_{\mathrm{L}}$ and $d A_{\mathrm{K}}$ separated by a distance $l$. The angle between the surface-to-surface vector and the normal to each surface element is $\psi_{\mathrm{L}}$ and $\psi_{\mathrm{K}}$. (b) two parallel infinite plates separated by a distance $H$.

Equating Eq (8) in flat parallel plates geometry, as shown in Fig 2b, and Eq (9) and solving for $e_{\mathrm{DLVO}}(1)$ results in,

$$
e_{D L V O}(l)=-\frac{l}{2 \pi} \frac{d}{d l} \frac{E_{D L V O}(l)}{l^{2}} .
$$

For the particular case of DLVO potentials, the relevant functions are

$$
E_{D L V O}(H)=32 \varepsilon \kappa\left(\frac{k T}{e}\right)^{2} \tanh \left(\frac{e \zeta_{L}}{4 k T}\right) \tanh \left(\frac{e \zeta_{K}}{4 k T}\right) \exp (-\kappa H) \text { and }
$$




$$
e_{D L V O}(l)=-\frac{16 \varepsilon \kappa}{\pi}\left(\frac{k T}{e}\right)^{2} \tanh \left(\frac{e \zeta_{L}}{4 k T}\right) \tanh \left(\frac{e \zeta_{K}}{4 k T}\right) \exp (-\kappa l)\left[\frac{\kappa}{l}+\frac{2}{l^{2}}\right] .
$$

The validity of equations (11) and (12) was tested and validated against expected results for a sphere interacting with a wall and for sphere-sphere interactions.

Hydrodynamic interactions for spheres

For creeping flow, the hydrodynamic drag force, $\mathbf{F}^{\mathrm{H}}$, and torque, $\mathbf{T}^{\mathrm{H}}$, on particle $i$ are related to particle translational, $\mathbf{U}_{i}$, and angular, $\Omega_{\square}$ velocities by

$$
\left(\begin{array}{c}
\mathbf{F}^{H}{ }_{L} \\
\mathbf{F}^{H}{ }_{K} \\
\mathbf{T}^{H}{ }_{L} \\
\mathbf{T}^{H}{ }_{K}
\end{array}\right)=\mathbf{R} \cdot\left(\begin{array}{c}
\mathbf{U}_{L} \\
\mathbf{U}_{K} \\
\Omega_{L} \\
\Omega_{K}
\end{array}\right)
$$

where $\mathbf{R}$ is the hydrodynamic resistance tensor, calculated using the arbitrary separation solution in the classic work of Jeffrey and Onishi[23, 24], which can be expanded to

$$
\mathbf{R}=\mu\left(\begin{array}{cccc}
\mathbf{A}_{L L} & \mathbf{A}_{L K} & \tilde{\mathbf{B}}_{L L} & \tilde{\mathbf{B}}_{L K} \\
\mathbf{A}_{K L} & \mathbf{A}_{K K} & \tilde{\mathbf{B}}_{K L} & \tilde{\mathbf{B}}_{K K} \\
\mathbf{B}_{L L} & \mathbf{B}_{L K} & \mathbf{C}_{L L} & \mathbf{C}_{L K} \\
\mathbf{B}_{K L} & \mathbf{B}_{K K} & \mathbf{C}_{K L} & \mathbf{C}_{K K}
\end{array}\right)
$$

where the A quadrant relates translational velocities with forces, the $\mathbf{C}$ quadrant relates angular velocities with torques, and $\mathbf{B}$ quadrants correlate translational and rotational motion, and $\mu$ is the solvent viscosity. Components of each sub tensor can be calculated using the following relations:

$$
\begin{gathered}
A_{i j}^{(\alpha \beta)}=X_{\alpha \beta}^{A} d_{i} d_{j}+Y_{\alpha \beta}^{A}\left(\delta_{i j}-d_{i} d_{j}\right), \\
B_{i j}^{(\alpha \beta)}=\tilde{B}_{j i}{ }^{(\alpha \beta)}=Y_{\alpha \beta}^{B} \varepsilon_{i j k} d_{k}, \text { and } \\
C_{i j}^{(\alpha \beta)}=X_{\alpha \beta}^{C} d_{i} d_{j}+Y_{\alpha \beta}^{C}\left(\delta_{i j}-d_{i} d_{j}\right),
\end{gathered}
$$

where the subscripts $i$ and $j$ denote direction in Cartesian coordinates, $\alpha$ and $\beta$ denote particle identity (i.e. lock or key), $\mathbf{d}$ is the unit vector between particle centers $(\mathbf{d}=\mathbf{r} /|\mathbf{r}|)$, $\varepsilon_{\mathrm{ijk}}$ is the permutation operator, and the functions $X^{\mathrm{A}}{ }_{\alpha \beta}, Y_{\alpha \beta}^{\mathrm{A}}, Y^{\mathrm{B}}{ }_{\alpha \beta}, X^{\mathrm{C}}{ }_{\alpha \beta}$, and $Y_{\alpha \beta}^{\mathrm{C}}$ are the arbitrary separation solutions given by Jeffrey and Onishi[23, 24].

\section{Smoluchowski collision rate modeling of $F$ to $S$ and NS transitions}

The transition rate of free to bound (either specific or non-specific) configurations can be estimated by the collision rate of lock and key particles, $k_{\mathrm{F}}$, and is given from an expression of 
Smoluchowski by[14, 19]

$$
k_{F}=\frac{2 k T}{3 \mu}\left(\frac{1}{a_{L}}+\frac{1}{a_{K}}\right)\left(a_{L}+a_{K}\right),
$$

where $\mu$ is the solvent viscosity. To calculate the transition rate of free to specific and free to non-specific configurations, the collision rate is weighted by the fractional surface area of the lock that corresponds to either specific (i.e. the fraction of lock surface area occupied by the dimple), $f$, or non-specific, $1-f$, as

$$
f=\frac{1-\cos \alpha}{2},
$$

where $\mathrm{a}$ is the lock aperture angle, as seen in Fig 1c, yielding

$$
\begin{gathered}
k_{F-S}=f k_{F}, \text { and } \\
k_{F-N S}=(1-f) k_{F} .
\end{gathered}
$$

\section{Diffusion-migration modeling of NS to $S$ and $F$ transitions}

To model the kinetics of transitions from non-specific configurations to other configurations we use a diffusion-migration description[14, 25] of lock and key particle pair motion in terms of relative surface-to-surface separation, $h$, and relative orientation angle, $\theta$, as specified in Fig 1c. The evolution of probability density, $\rho(h, \theta, t)$, is modeled using the Smoluchowski equation,

$$
\frac{\partial \rho(h, \theta, t)}{\partial t}=\nabla \cdot \mathbf{D}(h, \theta) \exp \left[-\frac{W(h, \theta)}{k T}\right] \cdot \nabla \exp \left[\frac{W(h, \theta)}{k T}\right] \rho(h, \theta, t),
$$

where $W(h, \theta)$ is a potential of mean-force, explained below, and $\mathbf{D}(h, \theta)$ is a diffusion tensor related to hydrodynamic interactions between lock and key in normal $(h)$ and tangential $(\theta)$ directions, also explained below. Equation (22) can be written in terms of the probability current, $\mathbf{S}$, as

$$
\frac{\partial \rho(h, \theta, t)}{\partial t}=-\nabla \cdot \mathbf{S}(h, \theta) .
$$

Equation (22) is solved with a Boltzmann-weighted initial condition based on the potential of mean-force,

$$
\rho(h, \theta, 0) \propto \exp \left[-\frac{W(h, \theta)}{k T}\right],
$$

in the non-specific region of the $h-\theta$ phase space, defined as $h<h_{\mathrm{a}}$, and $\theta>\alpha$. The boundary conditions are absorbing at $h=h_{\mathrm{a}}$, signifying a transition between non-specific and free configurations, and at $\theta=\alpha$, signifying a transition between non-specific and specific 
configurations, as seen in Fig 1c.

Potential of mean force

The potential of mean-force, $W(h, \theta)$, is calculated as[14]

$$
W(h, \theta)=\phi(h, \theta)+W_{g}(h, \theta),
$$

where $\phi(h, \theta)$ is the interaction potential, as explained above and $W_{\mathrm{g}}(h, \theta)$ is a configurational contribution resulting from the particular choice of coordinates as

$$
W_{g}(h, \theta)=-2 k T \ln \left(h+a_{L}+a_{K}\right)-k T \ln (\sin \theta)+C,
$$

and $C$ is an arbitrary constant. The first term on the right hand side of Eq (26) is related to the higher degeneracy of states at higher particle separations, while the second term is related to the higher degeneracy of states for configurations where the key is closer to the equator of the lock. The Supplementary Material shows the derivation of Eq. (26).

\section{Diffusivity from hydrodynamic interactions}

The diffusivity tensor in Eq (22) is related to components of the hydrodynamic mobility tensor, $\mathbf{M}=\mathbf{R}^{-1}$, constructed with mobility functions $x_{\alpha \beta}^{\mathrm{a}}, y_{\alpha \beta}^{\mathrm{a}}, y_{\alpha \beta}^{\mathrm{b}}, x_{\alpha \beta}^{\mathrm{c}}$, and $y_{\alpha \beta}^{\mathrm{c}}$ equivalent to the resistance functions $X_{\alpha \beta}^{\mathrm{A}}, Y_{\alpha \beta}^{\mathrm{A}}, Y_{\alpha \beta}^{\mathrm{B}}, X_{\alpha \beta}^{\mathrm{C}}$, and $Y_{\alpha \beta}^{\mathrm{C}}$ from Eqs (15)-(17) and are estimated by inverting the resistance tensor, $\mathbf{R}$, in Eq (14). The particular components that make up the diffusion tensor, $\mathbf{D}$, needed for our problem with two degrees of freedom, are

$$
\mathbf{D}(h, \theta)=\frac{k T}{\mu}\left(\begin{array}{cc}
x^{a}{ }_{L L}+x^{a}{ }_{K K}-2 x^{a}{ }_{L K} & 0 \\
0 & y_{L L}^{c}+\frac{y_{L L}^{a}+y^{a}{ }_{K K}-2 y^{a}{ }_{L K}}{\left(h+a_{L}+a_{K}\right)^{2}}+2 \frac{y_{L K}^{b}-y_{L L}^{b}}{\left(h+a_{L}+a_{K}\right)}
\end{array}\right) .
$$

See the Supplementary Material for the derivation of Eq (27).

First passage time distributions

The solution of Eq. (22) can be used to calculate first passage time distributions for nonspecific to free or specific, $w_{\mathrm{NS}-\mathrm{B}}(t)$ (B being $\mathrm{F}$ or $\left.\mathrm{S}\right)$, events as

$$
w_{N S-B}(t)=\int_{\mathscr{S}_{N S-H}} \mathbf{S}(h, \theta, t) \cdot d \mathbf{n}^{\prime},
$$

where the integral is evaluated over the boundary $\mathrm{S}_{\mathrm{NS}-\mathrm{B}}$, between states NS and $\mathrm{B}$, and $\mathrm{dn}$ ' is a vector normal to the boundary. From first passage time distributions we calculate the zeroth moment,

$$
P_{N S-B}=\int_{0}^{\infty} w_{N S-B}(t) d t,
$$

which is the probability that the system starting in a non-specific configuration transitions to state B before transitioning to the complementary state. The zeroth moment for NS to S events is called the success probability, $P_{\mathrm{S}}$. Normalization of $w_{\mathrm{NS}-\mathrm{B}}(t)$ by its zeroth moment, 


$$
p_{N S-B}=\frac{w_{N S-B}(t)}{P_{N S-B}},
$$

is the first passage time distribution conditional to events of the same type. The first moment of $p_{\mathrm{NS}-\mathrm{B}}(t)$,

$$
\tau_{M F P, N S-B}=\int_{0}^{\infty} p_{N S-B}(t) t d t
$$

is the mean first passage time of events of this kind.

The first passage time for all events starting from non-specific configurations is given by

$$
\tau_{M F P, N S}=\int_{0}^{\infty}\left(w_{N S-F}(t)+w_{N S-S}(t)\right) t d t .
$$

The kinetic rate constant for events starting in non-specific configurations is

$$
k_{N S-B}=\frac{P_{N S-B}}{\tau_{M F P, N S-B}} .
$$

\section{Equilibrium thermodynamics of transitions between states}

The rates at which transitions occur between the states shown in Fig 1a are given by[14]

$$
\begin{gathered}
r_{F-S}=k_{F-S} n_{L} n_{K}, \\
r_{F-N S}=k_{F-N S} n_{L} n_{K}, \\
r_{N S-S}=k_{N S-S} n_{N S}, \\
r_{N S-F}=k_{N S-F} n_{N S}, \\
r_{S-N S}=k_{S-N S} n_{S}, \text { and } \\
r_{S-F}=k_{S-F} n_{S},
\end{gathered}
$$

where $n_{\mathrm{L}}, n_{\mathrm{K}}, n_{\mathrm{NS}}$, and $n_{\mathrm{S}}$ are concentrations of lock particles, key particles, non-specific pairs and specific pairs, respectively, $r_{\mathrm{F}-\mathrm{S}}, r_{\mathrm{F}-\mathrm{NS}}, r_{\mathrm{NS}-\mathrm{S}}, r_{\mathrm{NS}-\mathrm{F}}, r_{\mathrm{S}-\mathrm{NS}}$, and $r_{\mathrm{S}-\mathrm{F}}$ are rates per unit volume of free to specific, free to non-specific, non-specific to specific, non-specific to free, specific to non-specific, and specific to free, respectively, events. Rate constants determined from different methodologies are be related to the equilibrium behavior of the reactions involved. At equilibrium, association and dissociation rates between non-specific, free, and specific configurations are equal, yielding the relations

$$
\begin{aligned}
& k_{F-N S} n_{L} n_{K}=k_{N S-F} n_{N S}, \\
& k_{F-S} n_{L} n_{K}=k_{S-F} n_{S}, \text { and }
\end{aligned}
$$




$$
k_{S-N S} n_{S}=k_{N S-S} n_{N S} .
$$

A combination of Eqs. (40) to (42) yields

$$
\frac{k_{N S-S}}{k_{S-N S}}=\frac{k_{N S-F} k_{F-S}}{k_{F-N S} k_{S-F}} .
$$

which serves as a thermodynamic consistency check for estimations and measurements of kinetic rate constants[14]. Additionally, ratios of kinetic rate constants, in particular Eq. (42), can be related to free energy differences between non-specific and specific configurations, $\Delta F_{\mathrm{NS}-\mathrm{F}}$, as

$$
\Delta F_{N S-S}=-k T \ln \left(\frac{k_{N S-S}}{k_{S-N S}}\right) .
$$

The free energy between non-specific and specific configurations can also be calculated from the general shape interaction potentials described previously, namely

$$
\Delta F_{N S-S}=k T \ln \left(\frac{\int_{V_{N S}} d V \exp [-\phi(r, \theta)]}{\int_{V_{S}} d V \exp [-\phi(r, \theta)]}\right),
$$

where the integration volumes $V_{\mathrm{NS}}$ and $V_{\mathrm{S}}$ correspond to non-specific and specific regions as shown in Fig 1c and explained previously. Equations (44) and (45) provide a method to estimate the specific to non-specific kinetic rate constant, $k_{\mathrm{S}-\mathrm{NS}}$ from diffusion-migration model calculations and the general shape interaction potential,

$$
k_{S-N S}=k_{N S-S}\left(\frac{\int_{W_{N S}} d V \exp [-\phi(r, \theta)]}{\int_{V_{S}} d V \exp [-\phi(r, \theta)]}\right) .
$$

Finally, by estimating kinetic rate constants for free to specific and non-specific (F-S/NS) from Smoluchowski collision rates (given in Eqs. (20) and (21)), non-specific to specific and free (NS-S/F) from diffusion-migration calculations, specific to non-specific (S-NS) using Eq. (46), and using these results with the thermodynamic consistency, Eq. (43), the specific to free (S-F) kinetic rate constant can be estimated as

$$
k_{S-F}=\frac{k_{S-N S} k_{N S-F} k_{F-S}}{k_{N S-S} k_{F-N S}} .
$$




\section{Methods}

\section{Diffusion-migration model solution}

To solve the diffusion-migration model we use non-specific potentials, given in Eq. (1), for the calculation of the potential of mean force, $W(h, \theta)$, in Eq. (25), and we use sphere-sphere hydrodynamic interactions for the calculation of the diffusivity tensor, $\mathbf{D}(h)$, in Eq. (27). The rationale behind this simplified model is that binding (non-specific to specific transition) is assumed to occur fast once the key reaches an orientation angle $\theta=\alpha$, so most details of the lock shape are outside the integration domain of the diffusion-migration model. Possible sources of error include the effect of a "depletion barrier"[21] (a region where depletion potentials become weak near the lip of the lock) which was addressed in a previous publication[14] and is readdressed in our results here, and a change in hydrodynamic interactions near the complex geometry of the dimple which is minimized by focusing the model on the spherical surface of the lock. A more thorough treatment of the hydrodynamic interactions between arbitrary shapes and its inclusion in the diffusion-migration model is beyond the scope of this article, but, as shown in a previous publication[14], inclusion of detailed hydrodynamics is not essential as the simplified sphere-sphere calculations agree with experimental measurements. Figure 3 shows the relevant input functions for the diffusion-migration model based on non-specific interactions and spheresphere hydrodynamic interactions. It is worth to notice that $\phi^{\mathrm{NS}}$ and $\mathbf{D}$ are functions of $h$ alone.

Equation (22) is solved numerically by converting the continuous Smoluchowski equation to an equivalent discrete approximation given by a master equation of the form[26, 27]

$$
\begin{array}{r}
\frac{d p_{i}(t)}{d t}=\frac{d p\left(h_{l}, \theta_{m}, t\right)}{d t}=-\left[\begin{array}{l}
Q\left(h_{l+1}, \theta_{m} \mid h_{l}, \theta_{m}\right)+Q\left(h_{l}, \theta_{m+1} \mid h_{l}, \theta_{m}\right)+ \\
Q\left(h_{l-1}, \theta_{m} \mid h_{l}, \theta_{m}\right)+Q\left(h_{l}, \theta_{m-1} \mid h_{l}, \theta_{m}\right)
\end{array}\right] p\left(h_{l}, \theta_{m}, t\right)+ \\
Q\left(h_{l}, \theta_{m} \mid h_{l+1}, \theta_{m}\right) p\left(h_{l+1}, \theta_{m}, t\right)+Q\left(h_{l}, \theta_{m} \mid h_{l-1}, \theta_{m}\right) p\left(h_{l-1}, \theta_{m}, t\right)+ \\
Q\left(h_{l}, \theta_{m} \mid h_{l}, \theta_{m+1}\right) p\left(h_{l}, \theta_{m+1}, t\right)+Q\left(h_{l}, \theta_{m} \mid h_{l}, \theta_{m-1}\right) p\left(h_{l}, \theta_{m-1}, t\right)
\end{array}
$$

where $p\left(h_{1}, \theta_{\mathrm{m}}, t\right)$ is the probability of finding the system in a region of size $\Delta h \Delta \theta$ around the point $\left(h_{1}, \theta_{\mathrm{m}}\right)$ at time $t$, and the elements of the tensor, $\mathbf{Q}$, express probability transition rates between adjacent regions. Equation (48) can be expressed in short notation as

$$
\frac{d \mathbf{p}(t)}{d t}=\mathbf{Q} \cdot \mathbf{p}(t)
$$

where the discretized space is enumerated in a total of $N_{\mathrm{b}}$ bins, and the $i$ th component of the vector $\mathbf{p}$ is the probability $p\left(h_{1}, \theta_{\mathrm{m}}, t\right)$ around the point " $i$ ". 


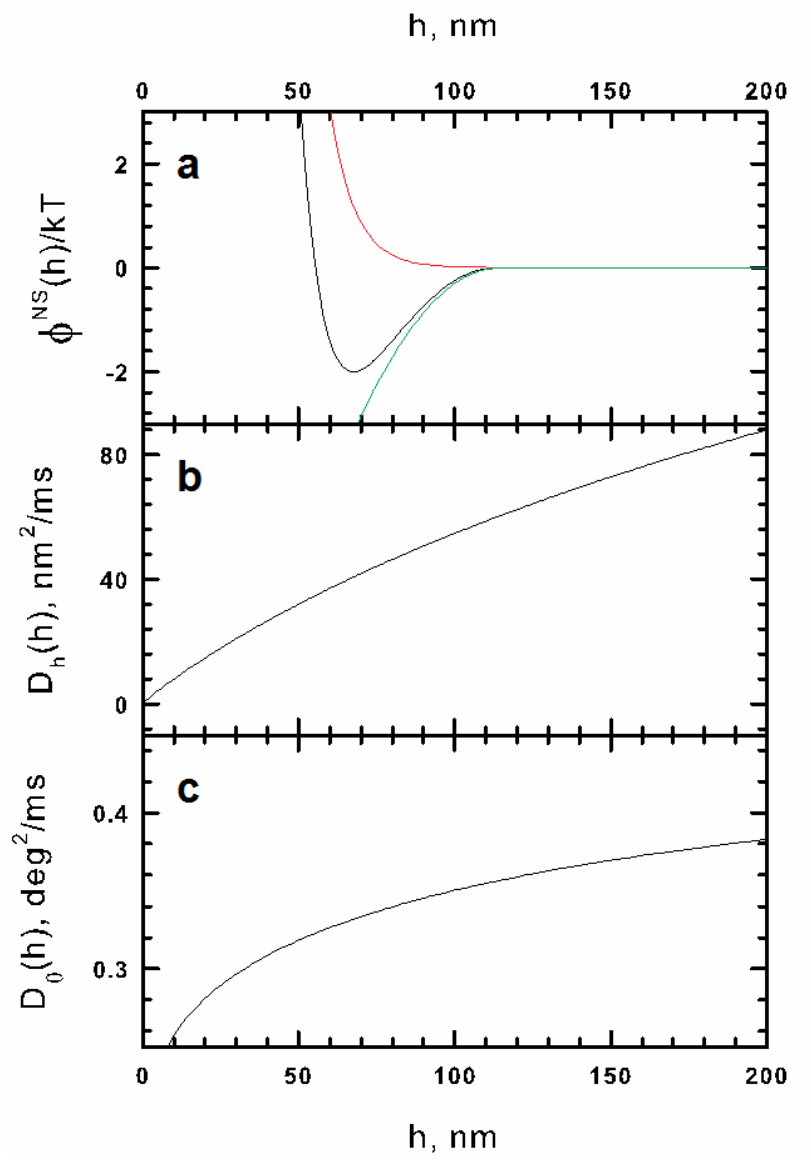

Fig 3. Non-specific potential energy and diffusivities. (a) potential energy, $\phi \square(h)$, between two unequal spheres, black line, comprised of a short-ranged electrostatic repulsion, red line, and a longer-ranged depletion attraction, green line. (b) effective diffusivity in the " $h$ " direction, $D_{\mathrm{h}}(h)$. (c) effective diffusivity in the " $\theta$ ' direction, $D_{\theta}(h)$.

\section{Numerical solution of the effective master equation}

The components of $\mathbf{Q}$ are related to both the potential of mean force and diffusivity tensor as

$$
\begin{aligned}
& Q\left(h_{l+1}, \theta_{m} \mid h_{l}, \theta_{m}\right)=-\frac{D_{h}\left(h_{l}, \theta_{m}\right)}{\Delta h^{2}} \frac{\left(W\left(h_{l+1}, \theta_{m}\right)-W\left(h_{l}, \theta_{m}\right)\right) / k T}{\exp \left[-\left(W\left(h_{l+1}, \theta_{m}\right)-W\left(h_{l}, \theta_{m}\right)\right) / k T\right]-1}, \\
& Q\left(h_{l}, \theta_{m} \mid h_{l+1}, \theta_{m}\right)=-\frac{D_{h}\left(h_{l}, \theta_{m}\right)}{\Delta h^{2}} \frac{\left(W\left(h_{l+1}, \theta_{m}\right)-W\left(h_{l}, \theta_{m}\right)\right) / k T}{\exp \left[\left(W\left(h_{l+1}, \theta_{m}\right)-W\left(h_{l}, \theta_{m}\right)\right) / k T\right]-1}, \\
& Q\left(h_{l}, \theta_{m+1} \mid h_{l}, \theta_{m}\right)=-\frac{D_{\theta}\left(h_{l}, \theta_{m}\right)}{\Delta \theta^{2}} \frac{\left(W\left(h_{l}, \theta_{m+1}\right)-W\left(h_{l}, \theta_{m}\right)\right) / k T}{\exp \left[-\left(W\left(h_{l}, \theta_{m+1}\right)-W\left(h_{l}, \theta_{m}\right)\right) / k T\right]-1},
\end{aligned}
$$




$$
Q\left(h_{l}, \theta_{m} \mid h_{l}, \theta_{m+1}\right)=-\frac{D_{\theta}\left(h_{l}, \theta_{m}\right)}{\Delta \theta^{2}} \frac{\left(W\left(h_{l}, \theta_{m+1}\right)-W\left(h_{l}, \theta_{m}\right)\right) / k T}{\exp \left[\left(W\left(h_{l}, \theta_{m+1}\right)-W\left(h_{l}, \theta_{m}\right)\right) / k T\right]-1},
$$

where $D_{\mathrm{h}}$ and $D_{\theta}$ are the " $h-h$ " and " $\theta-\theta$ ' components of $\mathbf{D}$, respectively. Note that the potential of mean force is given by Eq (25). Equation (49) can be solved numerically as

$$
\mathbf{p}(t+\Delta t)=\mathbf{Q} \cdot \mathbf{p}(t),
$$

where the integration time step, $\Delta t$, is $5 \times 10^{-3} \mathrm{~ms}$ (which is smaller than the particles characteristic translational, $\tau_{\mathrm{t}}=\Delta h^{2} / D_{\mathrm{h}}\left(h_{\mathrm{a}}\right) \sim 1 \mathrm{~ms}$, and rotational, $\tau_{\mathrm{r}}=\Delta \theta^{2} / D_{\theta}\left(h_{\mathrm{a}}\right) \sim 10 \mathrm{~ms}$, time scales based on the spatial resolution). The spatial resolution for the effective master equation is $\Delta h=10 \mathrm{~nm}$ and $\Delta \theta=0.035 \mathrm{rad}$, which are both small enough to resolve the potential of mean force. Both time integration step and spatial resolution values were tested against results with smaller values, yielding no significant differences in results. The initial condition for solving Eq (54) is the Boltzmann weighted probability distribution based on the potential of mean force given in Eq (24). In all cases considered the maximum $h$ (the maximum surface-to-surface distance between particles at which integration is done) is taken as $1000.0 \mathrm{~nm}$, which provides a solution that does not change with maximum distance. Equation (54) is integrated for a total of $20000.0 \mathrm{~ms}$, which provides a solution that does not change with total integration time. Table 1 provides information of additional parameters necessary for the solution of the effective master equation.

\section{Estimation of first passage time distributions}

To calculate first passage time distributions, $w_{\mathrm{NS}-\mathrm{B}}(t)$, from Eq. (28) we enforce absorbing boundary conditions (see the Theory section) at each time step by setting probabilities in the specific region $\left(h<h_{\mathrm{a}}, \theta<\alpha\right)$ and free region $\left(h \geq h_{\mathrm{a}}\right)$ to zero. The probability flow is calculated from the probabilities being set to zero in each application of the absorbing boundary conditions in each region (specific or free).

\section{Discrete sampling time solution}

Experimental measurements of transition rates for lock and key particle binding depend heavily on the temporal resolution of experiments[14]. To account for non-continuous time sampling we modify our absorbing boundary conditions. The non-specific to specific boundary is enforced each time step, to account for the fast binding once the key reaches the lip of the lock at $\theta=\alpha$, while the non-specific to free boundary is enforced every discrete sampling time, $\tau_{\mathrm{D}}$. This allows for probability to flow to the free region and come back to the non-specific region between absorbing boundary condition enforcing steps. First passage time distributions are then calculated with a resolution of $\tau_{\mathrm{D}}$.

\section{Stokesian dynamic (SD) simulations}

We generate microscopic configurations for a system comprised of a lock and a key particle in an unbounded medium by simulating the equations of motion[28, 29] given by 


$$
\left(\begin{array}{c}
\mathbf{r}_{L} \\
\mathbf{r}_{K} \\
\mathbf{n}_{L} \\
\mathbf{n}_{K}
\end{array}\right)_{(t+\Delta t)}=\left(\begin{array}{c}
\mathbf{r}_{L} \\
\mathbf{r}_{K} \\
\mathbf{n}_{L} \\
\mathbf{n}_{K}
\end{array}\right)_{(t)}+\Delta t\left(k T \nabla \cdot \mathbf{M}(t)+\mathbf{M}(t) \cdot\left(\begin{array}{c}
\mathbf{F}^{C}{ }_{L}+\mathbf{F}^{B}{ }_{L} \\
\mathbf{F}^{C}{ }_{K}+\mathbf{F}^{B}{ }_{K} \\
\left(\mathbf{T}_{L}^{C}+\mathbf{T}^{B}{ }_{L}\right) \times \mathbf{n}_{L} \\
\left(\mathbf{T}^{C}{ }_{K}+\mathbf{T}^{B}{ }_{K}\right) \times \mathbf{n}_{K}
\end{array}\right)_{(t)}\right),
$$

where $\Delta t$ is the integration time, $\mathbf{r}_{\mathrm{i}}$ and $\mathbf{n}_{\mathrm{i}}$ are the position and orientation vectors for particle $i$, respectively, $\mathbf{M}$ is the hydrodynamic mobility tensor, $\mathbf{F}^{\mathrm{C}}{ }_{\mathrm{i}}$ and $\mathbf{T}_{\mathrm{i}}^{\mathrm{C}}$ are conservative force and torque on particle $i$, and $\mathbf{F}^{\mathrm{B}}{ }_{\mathrm{i}}$ and $\mathbf{T}^{\mathrm{B}}{ }_{\mathrm{i}}$ are Brownian force and torque on particle $i$. To avoid the time-consuming computation of the gradient of the mobility tensor we use the mid-point algorithm[30,31] given by

$$
\begin{aligned}
& \left(\begin{array}{c}
\mathbf{r}_{L} \\
\mathbf{r}_{K} \\
\mathbf{n}_{L} \\
\mathbf{n}_{K}
\end{array}\right)^{*}=\left(\begin{array}{c}
\mathbf{r}_{L} \\
\mathbf{r}_{K} \\
\mathbf{n}_{L} \\
\mathbf{n}_{K}
\end{array}\right)_{(t)}+0.5 \Delta t \mathbf{M}(t) \cdot\left(\begin{array}{c}
\mathbf{F}_{L}^{C}+\mathbf{F}^{B}{ }_{L} \\
\mathbf{F}^{C}{ }_{K}+\mathbf{F}^{B}{ }_{K} \\
\left(\mathbf{T}^{C}{ }_{L}+\mathbf{T}^{B}{ }_{L}\right) \times \mathbf{n}_{L} \\
\left(\mathbf{T}^{C}{ }_{K}+\mathbf{T}^{B}{ }_{K}\right) \times \mathbf{n}_{K}
\end{array}\right)_{(t)} \text {, and } \\
& \left(\begin{array}{c}
\mathbf{r}_{L} \\
\mathbf{r}_{K} \\
\mathbf{n}_{L} \\
\mathbf{n}_{K}
\end{array}\right)_{(t+\Delta t)}=\left(\begin{array}{c}
\mathbf{r}_{L} \\
\mathbf{r}_{K} \\
\mathbf{n}_{L} \\
\mathbf{n}_{K}
\end{array}\right)_{(t)}+\Delta t \mathbf{M}^{*} \cdot\left[\left(\begin{array}{c}
\mathbf{F}^{C}{ }_{L} \\
\mathbf{F}^{C}{ }_{K} \\
\mathbf{T}^{C}{ }_{L} \times \mathbf{n}_{L} \\
\mathbf{T}^{C}{ }_{K} \times \mathbf{n}_{K}
\end{array}\right)^{*}+\left(\begin{array}{c}
\mathbf{F}^{B}{ }_{L} \\
\mathbf{F}^{B}{ }_{K} \\
\mathbf{T}^{B}{ }_{L} \times \mathbf{n}_{L} \\
\mathbf{T}^{B}{ }_{K} \times \mathbf{n}_{K}
\end{array}\right)_{(t)}\right],
\end{aligned}
$$

where quantities with a star superscript denote calculations made at the mid-point configuration $\mathbf{r}_{i}{ }^{*}, \mathbf{n}_{\mathrm{i}}{ }^{*}$. Conservative forces are calculated by taking the negative gradient of the interaction potential, $\phi$, as

$$
\mathbf{F}^{C}=-\nabla \phi .
$$

Conservative torques are calculated as

$$
\mathbf{T}^{C}=\mathbf{r} \times \mathbf{F}^{C},
$$

where $\mathbf{r}$ is the center-to-center vector between particles. Brownian forces and torques are related to the hydrodynamic resistance tensor by the fluctuation-dissipation theorem[32] and have the following properties: 


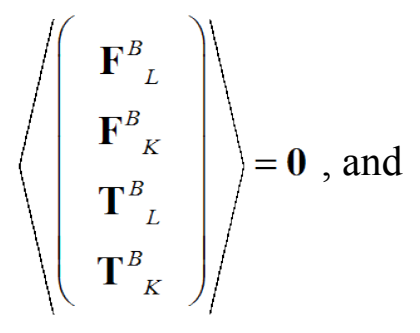

$$
\left.\int\left(\begin{array}{c}
\mathbf{F}^{B}{ }_{L} \\
\mathbf{F}^{B}{ }_{K} \\
\mathbf{T}^{B}{ }_{L} \\
\mathbf{T}^{B}{ }_{K}
\end{array}\right)_{(t)}\left(\begin{array}{c}
\mathbf{F}^{B}{ }_{L} \\
\mathbf{F}^{B}{ }_{K} \\
\mathbf{T}^{B}{ }_{L} \\
\mathbf{T}^{B}{ }_{K}
\end{array}\right)_{(t+\Delta t)}\right)=2 k T \mathbf{R} \delta(\Delta t),
$$

where $\delta(\Delta t)$ is the Dirac delta function.

Stokesian dynamic simulations are run at the same conditions as the diffusion-migration model to corroborate model results. The integration time step for Stokesian dynamic simulations is $\Delta t=5 \times 10^{-2} \mathrm{~ms}$, and additional simulation details are contained in Table 1 .

\section{Estimation of general shape interaction potentials}

Figure 4a shows a TEM micrograph of a sample lock particle that is approximated by 3 distinct curvature regions: (1) the lock surface, of radius $a_{\mathrm{L}}=1200 \mathrm{~nm}$, (2) the lock lip, of radius $a_{\mathrm{LL}}=150 \mathrm{~nm}$, and (3) the lock dimple of radius $a_{\mathrm{LD}}=967 \mathrm{~nm}$. The angle of dimple aperture, $\alpha$, is defined as $\alpha=\tan ^{-1} z_{\mathrm{L}} / x_{\mathrm{L}}=0.68 \mathrm{rad}=39^{\circ}$, where $z_{\mathrm{L}}=813 \mathrm{~nm}$ and $x_{\mathrm{L}}=664 \mathrm{~nm}$ are the relative coordinates to the center of the lip. The center of the dimple curvature lies at a distance $a_{\mathrm{LD}}+z_{\mathrm{D}}=1712 \mathrm{~nm}$ from the center of the lock.

\section{Depletion potential}

To calculate the overlap excluded volume for general shape interaction, $\Delta V(r, \theta)$, in Eq. (7) we perform numerical integration of the volume shared by two overlapping bodies (a lock and a key both augmented by the radius of depletants, as specified in Table 1), seen schematically in Fig $1 \mathrm{~b}$. The spatial resolution for this integration is $\mathrm{d} x=\mathrm{d} y=\mathrm{d} z=1 \mathrm{~nm}=8.33 \times 10^{-}$ ${ }^{4} a_{\mathrm{L}}$. The calculation is made on a grid of relative positions between lock and key $(r, \theta)$ with a resolution of $\mathrm{d} r=1 \mathrm{~nm}=8.33 \times 10^{-4} a_{\mathrm{L}}$ and $\mathrm{d} \theta=1.75 \times 10^{-2} \mathrm{rad}$. Figure $4 \mathrm{~b}$ shows the results for $\Delta V(r, \theta)$ around the dimple, lip and surface of the lock.

\section{DLVO potential}

The calculation of arbitrary shape DLVO potential, $\phi_{\mathrm{DLVO}}(r, \theta)$, following Eq. (8) is made by constructing a grid on spherical coordinate angles on both lock and key and performing calculations on unit surface areas delimited by the angle grid. The azimuth, $\phi^{\prime}$, and elevation, $\theta$, angle grid resolution is $\mathrm{d} \phi^{\prime}=\mathrm{d} \theta=1.75 \times 10^{-2} \mathrm{rad}$. The cutoff distance for calculations (the distance at which potential contributions are deemed to be too small to contribute to the integral) is taken to be $15.0 \kappa^{-1}$. Figure $3 \mathrm{c}$ shows the results of $\phi_{\mathrm{DLVO}}(r, \theta)$ for the same regions shown in Fig $4 \mathrm{c}$ for conditions shown in Table 1. 

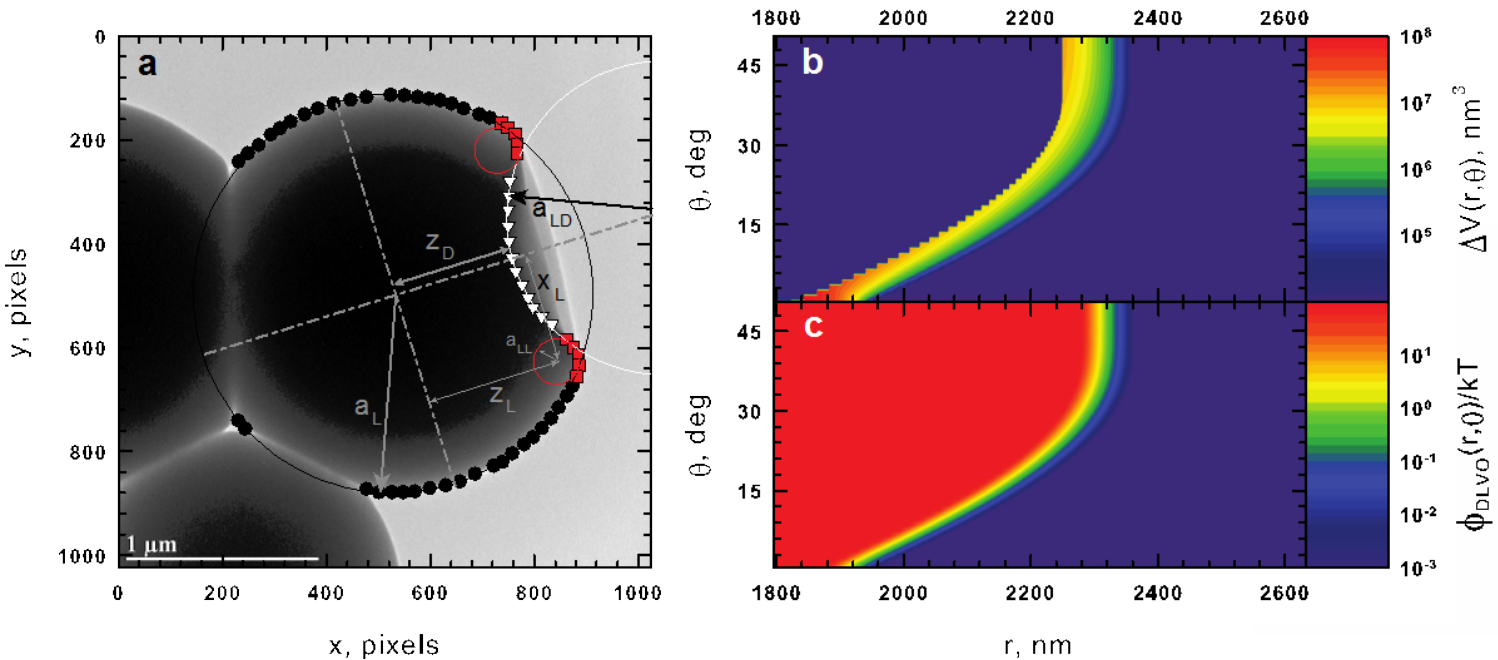

Fig 4. Lock geometry and overall lock-key potential. (a) Lock particle TEM micrograph and important geometry parameters. Three radii of curvature approximate the lock shape: lock surface radius, $a_{\mathrm{L}}$, lock lip radius, $a_{\mathrm{LL}}$, and lock dimple radius, $a_{\mathrm{LD}}$. Other parameters include the relative position of the lip center, $x_{\mathrm{L}}$, $z_{\mathrm{L}}$, and lock-center-to-dimple-basin distance, $z_{\mathrm{D}}$. (b) overlap excluded volume, $\Delta V$, as function of center-tocenter distance, $r$, and $\theta$ for the geometry shown in panel (a). (c) electrostatic potential calculated using Eq. (8) for the geometry shown in panel (a) for $\zeta \square=-80 \mathrm{mV}$ and $\kappa^{-1}=7.9 \mathrm{~nm}$.

\begin{tabular}{|c|c|}
\hline Parameter & Value \\
\hline$a_{\mathrm{L}}(\mathrm{nm})^{\mathrm{a}}$ & 1200 \\
\hline$a_{\mathrm{K}}(\mathrm{nm})^{\mathrm{b}}$ & 1050 \\
\hline$a_{\mathrm{D}}(\mathrm{nm})^{\mathrm{c}}$ & 57 \\
\hline$a_{\mathrm{LD}}(\mathrm{nm})^{\mathrm{d}}$ & 967 \\
\hline$a_{\mathrm{LL}}(\mathrm{nm})^{\mathrm{e}}$ & 150 \\
\hline$\alpha(\mathrm{deg})^{\mathrm{f}}$ & 39 \\
\hline$\varepsilon(\mathrm{F} / \mathrm{m})^{\mathrm{g}}$ & $7.08 \times 10^{-10}$ \\
\hline$\zeta_{\square} \zeta_{\mathrm{K}}(\mathrm{mV})^{\mathrm{h}}$ & -80.0 \\
\hline$\kappa^{-1}(\mathrm{~nm})^{\mathrm{i}}$ & 7.9 \\
\hline$\mu \square \mathrm{Pa} \mathrm{s} \dot{\mathrm{j}}$ & $8.94 \times 10^{-4}$ \\
\hline
\end{tabular}

Table 1. Relevant parameters for diffusion-migration model and Stokesian dynamic simulations. ${ }^{a}$ Lock particle radius. ${ }^{b}$ key particle radius. ${ }^{c}$ depletant radius. ${ }^{d}$ lock dimple radius for the TEM micrograph shown in Fig 4a. ${ }^{\mathrm{e}}$ lock lip radius for the TEM micrograph shown in Fig $4 \mathrm{a} .{ }^{\mathrm{f}}$ lock aperture angle, defined as $\alpha=$ $\tan ^{-1} x_{\mathrm{L}} / z_{\mathrm{L}}$ for the TEM micrograph shown in Fig 4a. ${ }^{\mathrm{g}}$ dielectric permittivity of solvent (water). ${ }^{\mathrm{h}}$ lock and key particle zeta potential. ${ }^{\mathrm{i}}$ Debye screening length. ${ }^{\mathrm{j}}$ solvent viscosity (water).

\section{Results \& Discussion}

\section{Lock and key thermodynamic-kinetic modeling assembly predictions}

In this section we look at predictions made with our modeling approach. First we address 
the predictions made with the diffusion-migration model to study the competing non-specific to specific and free configurations to understand the effect of the non-specific interaction strength on predicted kinetics. We proceed to study the effect of lock particle dimple and key particle shape complementarity on the stability of the specific state and escape, i.e. specific to nonspecific and free, kinetics. We finalize the section by constructing a design chart to guide the formation of stable specific pairs based on our predictions on both specific pair stability and kinetic transition rates.

\section{Diffusion-migration model predictions and the effect of NS potential energy well depth on NS to} $F$ and NS to S kinetics

We start our discussion by studying the nature of the competing non-specific to specific and free configurations dictated by the diffusion-migration framework developed in the Theory section. Figure 5 shows the kinetic properties of non-specific to free and specific events for a lock aperture angle $\alpha=30^{\circ}$, escape distance $h_{\mathrm{a}}=300 \mathrm{~nm}\left(0.25 a_{\mathrm{L}}\right)$, instantaneous sampling time $\left(\tau_{\mathrm{D}}\right.$ $\rightarrow 0$ ), and non-specific potential energy minimum varying from $\phi^{\mathrm{NS}}{ }_{\min }=1 k T$ to $4 k T$. Fig 5a shows non-specific to free first passage time distributions, $p_{\mathrm{NS}-\mathrm{F}}(t)$, while Fig $5 \mathrm{~b}$ shows non-specific to specific first passage time distributions, $p_{\mathrm{NS}-\mathrm{S}}(t)$. The success probability, as specified in the theory section, is reported in Fig $5 \mathrm{c}$ as a function of $\phi^{\mathrm{NS}}{ }_{\text {min }}$. The kinetic rate constants for nonspecific to free and non-specific to specific transitions, as functions of $\phi^{\mathrm{NS}}{ }_{\text {min }}$, are shown in Fig $5 \mathrm{~d}$. Figure $5 \mathrm{e}$ shows mean first passage times, $\tau_{\mathrm{MFP}}$, as a function of $\phi^{\mathrm{NS}}{ }_{\text {min }}$ for non-specific to specific and non-specific to free events. We show in Fig 5e, additionally, estimations for the mean escape time between two spheres and mean first passage time for NS-S transitions with a very deep non-specific potential energy minimum. Figures $5 \mathrm{f}$ and $5 \mathrm{~g}$ show, respectively, success probabilities and mean first passage times for NS-F and NS-S transitions that are angle-resolved, that is, results depend on initial angle, $\theta_{0}$.

Stokesian dynamic simulation results compare very favorably with diffusion-migration model results in both first passage time distributions and success probabilities for all cases studied, which prove the feasibility of the method. One thing worth mentioning is that even though the information obtained from the diffusion-migration model can be acquired from the more traditional Stokesian dynamic simulations, the seemingly more intricate diffusionmigration model works much faster as the overall dimensionality of the problem is reduced (from 6 translational and 6 orientational degrees of freedom to only 2: $\theta$ and $h$ ) and the model provides ensemble statistics directly, rather than the multiple runs required for Stokesian dynamic realizations.

The effect of a deeper potential energy well for non-specific interactions results in slower transition times for both non-specific to free and non-specific to specific events, as seen in Figs $5 \mathrm{a}$ and $5 \mathrm{~b}$. At the same time, success probabilities, that is the probability that a non-specific pair will transition to specific before transitioning to free, increase with $\phi^{\mathrm{NS}}{ }_{\text {min }}$, as seen in Fig 5c. The overall effect, as seen in Fig $5 \mathrm{~d}$, is a net decrease in transition kinetic constants for NS-F events as $\phi_{\text {min }}^{\mathrm{NS}}$ increases, while NS-S kinetic constants remain stable. NS to $\mathrm{F}$ transition kinetics decrease because of the longer time NS pairs remain bound before key particles can escape the potential energy well. NS to $\mathrm{S}$ transition kinetics are not affected too much because the underlying process is surface diffusion, which does not depend on the strength of the NS binding potential. 

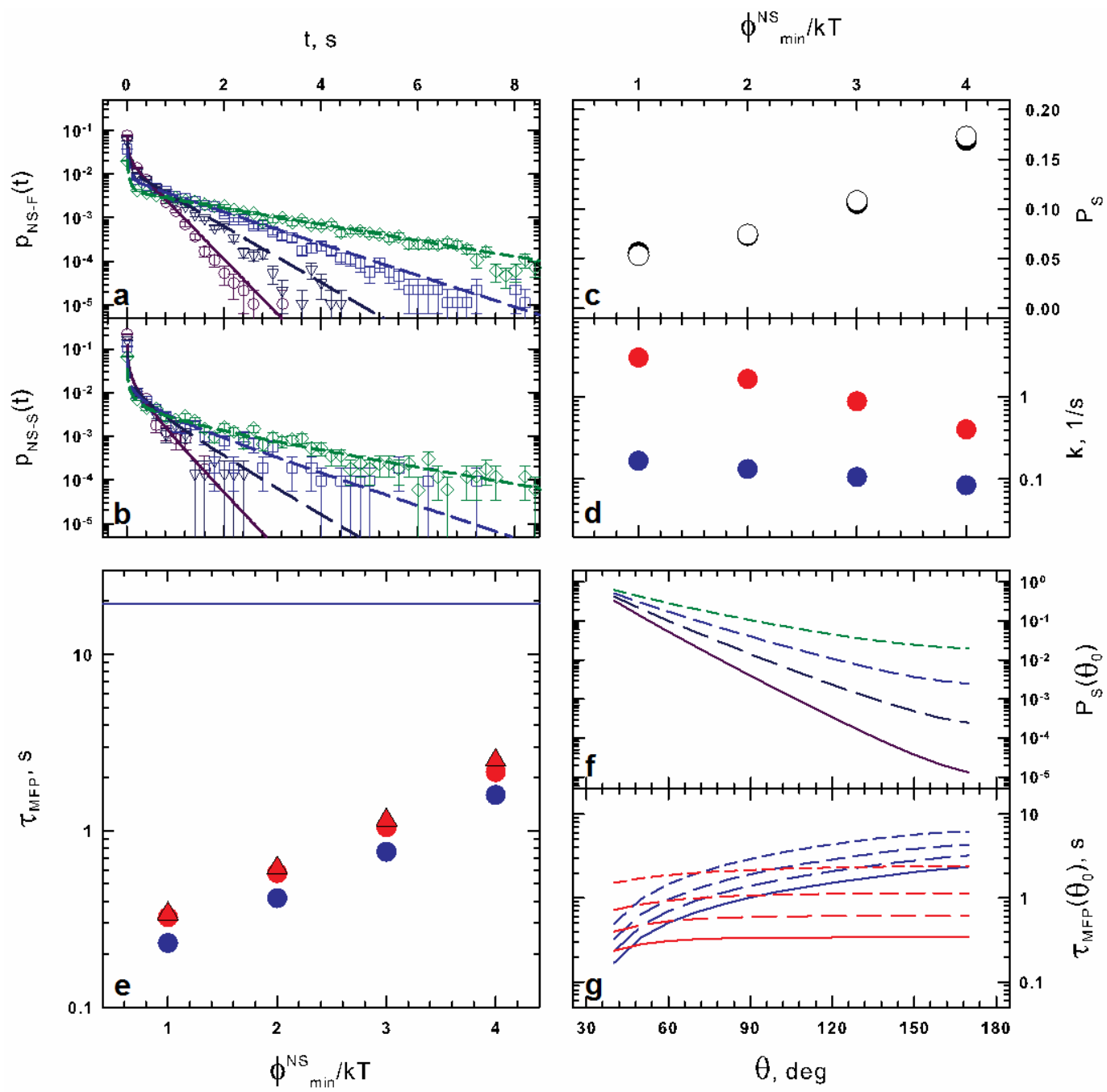

Fig 5. Effect of non-specific potential energy minimum, $\phi \square_{\text {min }}$, on predicted dynamics. (a) NS to F first passage time distribution, $p_{\text {NS-F }}$, lines denote diffusion-migration model results, symbols denote simulation results for $h_{\mathrm{a}}=300 \mathrm{~nm}, \alpha=30^{\circ}, \phi \square_{\min }=1 k T$ (purple, circles), $\phi \square_{\min }=2 k T$ (dark blue, triangles), $\phi \square_{\min }=3 k T$ (blue, squares), and $\phi D_{\min }=4 k T$ (green, diamonds). (b) NS to $\mathrm{S}$ first passage time distribution, $p_{\mathrm{NS}-\mathrm{s}}$, for the same cases shown in panel (a). (c) success probabilities, $P_{\mathrm{S}}$, as function of $\phi{ }_{\text {min }}$ for the conditions explored in panels (a) and (b) for simulations (white data) and model (black data). (d) kinetic rate constants, $k_{\mathrm{NS}-\mathrm{F}}(\mathrm{red})$ and $k_{\mathrm{NS}-\mathrm{S}}$ (blue), as function of $\phi \square_{\text {min }}$ for the conditions explored in panels (a) and (b). (e) mean first passage time, $\tau_{\mathrm{MFP}}$, as a function of $\phi{ }_{\text {min }}$ for NS to F events (red circles), NS-S events (blue circles), two-sphere mean escape time (red triangles), and deep well NS-S case (blue reference line) for conditions explored in panels (a) and (b). (f) Initial angle-resolved success probability, $P_{\mathrm{S}}\left(\theta_{0}\right)$, as a function of initial angular position, $\theta_{0}$, for $\phi{ }_{\min }=1 k T$ (purple solid line), $\phi \Gamma_{\text {min }}=2 k T$ (dark blue long-dashed line), $\phi \square_{\text {min }}=3 k T$ (blue medium-dashed line), and $\phi \Gamma_{\text {min }}=4 k T$ (green short-dashed line) for conditions explored in panels (a) and (b). (g) Initial angle-resolved mean first passage time, $\tau_{\mathrm{MFP}}\left(\theta_{0}\right)$ for NS to F events (red lines) and NS to $\mathrm{S}$ events (blue lines) for $\phi \square_{\text {min }}=1 k T$ (solid lines), $\phi D_{\text {min }}=2 k T$ (long-dashed lines), $\phi \square_{\text {min }}=3 k T$ (medium-dashed lines), and $\phi \square_{\text {min }}=4 k T$ (short-dashed lines) for conditions explored in panels (a) and (b).

These results can be explained by the following rationale: non-specific to free events 
become slower as $\phi^{\mathrm{NS}}{ }_{\text {min }}$ increases, seen as having a higher $\tau_{\mathrm{MFP}, \mathrm{NS}-\mathrm{F}}$ shown Fig $5 \mathrm{e}$, given that the binding energy of NS pairs is higher. Non-specific to specific kinetics, if the pair cannot unbind to become free, is independent of $\phi^{\mathrm{NS}}{ }_{\text {min }}$, as is shown in the no-escape (no NS-F process) case in Fig $5 \mathrm{e}$, as the key will diffuse in the surface of the lock until the boundary to specific, $S_{\mathrm{NS}-\mathrm{S}}$ at $\theta=\alpha$, is reached, but it is also slower than the non-specific to free transition, seen in the onlyescape (no NS-S process) case in Fig 5e. The NS-F kinetics will dominate the joint process, as successful NS-S events will have to be as fast, if not faster, than NS-F events. This can also be

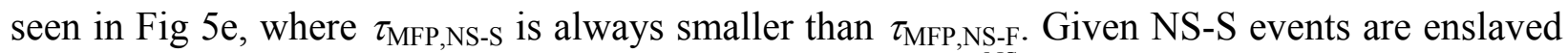
to NS-F events, as NS-F events become slower at higher $\phi^{\mathrm{NS}}{ }_{\min }$, so will NS-S events. Success probabilities increase because NS pairs remain bound longer, giving time to surface diffusion to turn NS pairs into S pairs.

To better understand how the NS to S and NS to F processes compete we look at angleresolved results shown in Figs $5 \mathrm{f}$ and $5 \mathrm{~g}$. The success probabilities shown in Fig $5 \mathrm{f}$ decrease significantly as the NS pair starts at a higher $\theta_{0}$, with the decrease being more evident in lower $\phi_{\text {min }}^{\mathrm{NS}}$ cases. This trend is expected, but it means that most of NS pairs become F, and only NS pairs where the key starts close to the lock lip, i.e. smaller $\theta_{0}$, have a decent chance of diffusing to the lock pocket. The lower success probabilities can also be related to higher mean NS to $\mathrm{S}$ event life times compared to NS to F events, i.e. $\tau_{\mathrm{MFP}, \mathrm{NS}-\mathrm{S}}>\tau_{\mathrm{MFP}, \mathrm{NS}-\mathrm{F}}$, at a given starting angle, as seen in Fig $5 \mathrm{~g}$. Both NS to F and NS to $\mathrm{S}$ mean event life times increase with increasing $\theta_{0}$ but NS to $\mathrm{F}$ event life times reach a plateau and remain fairly constant in almost all configurations. At low $\theta_{0}$ we see a crossover point, where NS to $\mathrm{S}$ events become faster than NS to $\mathrm{F}$, which is expected as the key can bind faster with the lock pocket than diffuse to a free configuration. We see that the larger the difference in mean event times, where NS to S events are slower than NS to $\mathrm{F}$ events, means a smaller success probability while in cases where NS to $\mathrm{S}$ events are faster than NS to $\mathrm{F}$ the success probability is higher.

Lock particle dimple and key particle shape complementarity effect on specific pair stability and escape kinetics

To study the effect of shape complementarity we calculate the free energy difference between non-specific and specific states, using Eq (45), for particles with different lock dimple radius, $a_{\mathrm{LD}}$, as shown in Figure $6 \mathrm{a}$ for fixed key particle, $a_{\mathrm{K}}$, radius as given in Table 1 . With free energy differences we calculate the specific to non-specific kinetic rate constant, $k_{\mathrm{S}-\mathrm{NS}}$, using Eq (46), where the non-specific to specific kinetic rate constant, $k_{\mathrm{NS}-\mathrm{S}}$, estimated using the diffusionmigration model using an escape distance $h_{\mathrm{a}}=300 \mathrm{~nm}$ and lock aperture angle $\alpha=30^{\circ}$, as shown in Fig 6b.

Free energy differences between NS and S states are shown to have a positive value for weak interaction strengths and switch to negative values after a crossover interaction strength, $\phi^{\mathrm{NS}}{ }_{\text {min,SS. At }} \phi^{\mathrm{NS}}{ }_{\min }>\phi^{\mathrm{NS}}{ }_{\text {min,SS }}$ specific binding is favored over non-specific binding, as $a_{\mathrm{LD}}$ changes we observe an optimum value for dimple radius that minimizes $\phi^{\mathrm{NS}}{ }_{\text {min,Ss. This optimum }}$ dimple size is not exactly the same size as the key radius, but slightly larger, which is to be expected, as a perfect fit will maximize the interaction potential energy, but the entropic cost to maintain the tight bond offsets the benefit. The optimal mismatch of lock particle dimple radius and key particle radius is consistent with similar findings in docking of ligands in enzymes[33]. Specific to non-specific kinetics are shown, in Fig 6b, to start at moderate values for small interaction strengths, but become vanishingly small for $\phi^{\mathrm{NS}}{ }_{\min }>\phi^{\mathrm{NS}}{ }_{\min , \mathrm{SS}}$. Confocal microscopy 
measurements of escape kinetics[14] show, nonetheless, that escape kinetic rate constants remain significant even at higher non-specific interaction strengths, as shown in the symbol data in Fig $6 \mathrm{~b}$, which in turn means that free energy differences, estimated from ratios of NS-S and S-NS kinetic rate constants in Eq (44), between specific and non-specific states as measured in the confocal microscopy study[14] (shown in symbols in Fig 6a) show a very different behavior, suggesting poorer shape complementarity between lock dimple and key. Experimental issues, related to the interpretation of data and assumptions inherent in the model will be discussed below.
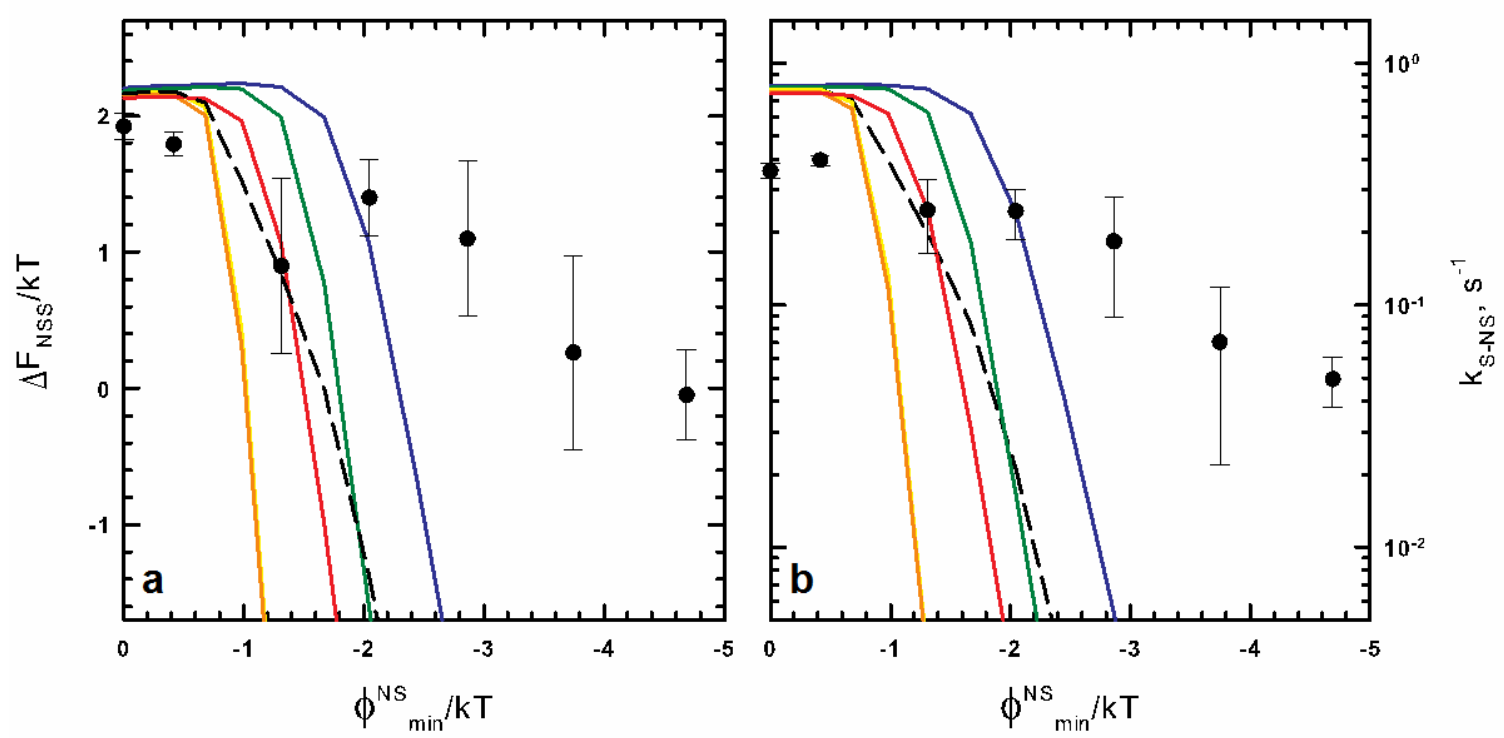

Fig 6. Lock geometry effect on NS to S selectivity. (a) NS to S free energy difference from Eq (45) as a function of minimum non-specific potential energy, $\phi \Gamma_{\text {min }}$, for average values of lock geometry parameters from Table 2 and $a_{\mathrm{LD}}=840 \mathrm{~nm}$ (blue), $a_{\mathrm{LD}}=940 \mathrm{~nm}$ (green), $a_{\mathrm{LD}}=1040 \mathrm{~nm}$ (yellow), $a_{\mathrm{LD}}=1140 \mathrm{~nm}$ (orange), and $a_{\mathrm{LD}}=1240 \mathrm{~nm}$ (red), as well as apparent free energy difference from Eq (63) (dashed black line) and experimental values from a previous confocal microscopy work[14] (symbols). (b) predicted $k_{\mathrm{S}-\mathrm{NS}}$ from Eq (46) as a function of $\phi{ }_{\text {min }}$ for the same cases in panel (a), weighted average, using Eq (62), from an $a_{\mathrm{LD}}$ polydispersity of $20 \%$ (dashed black line), and experimental values from a previous work[14] (symbols). Yellow and orange lines in panels (a) and (b) are almost juxtaposed.

\section{Designing colloidal lock-key particle assembly processes}

Estimates of kinetic rate constants, as explored in this work can serve to guide the design of assembly systems. In this section we summarize insights on the most relevant aspects of lockkey particle binding to pose a design chart for the rapid assembly of dumbbells, i.e. specific lockkey pairs. The first aspect to tackle is the working conditions that will form specific pairs and avoid undesirable structures. In the previous section we showed how a proper lock dimple size to key particle radius can have a impact on minimizing escape kinetics and ensure specific bonds are preferred over non-specific bonds. In Figure $7 \mathrm{a}$ we summarize these results in terms of the threshold non-specific potential energy minimum, $\phi^{\mathrm{NS}}{ }_{\mathrm{min}, \mathrm{SS}}$, as a function of lock dimple radius, $a_{\mathrm{LD}}$, for the geometries shown in Figs $4 \mathrm{a}$ and summarized in Table 1. As discussed before, we see an optimum value for the threshold interaction strength for a dimple radius larger than the 
lock radius. Threshold interaction strength increases away from the optimum value, indicating poorer shape recognition. To ensure specific binding one must work at interaction potentials stronger (i.e. more negative) than the threshold value, shown as a red tie-line between Fig $7 \mathrm{a}$ and $7 \mathrm{~b}$, for a given lock dimple radius.
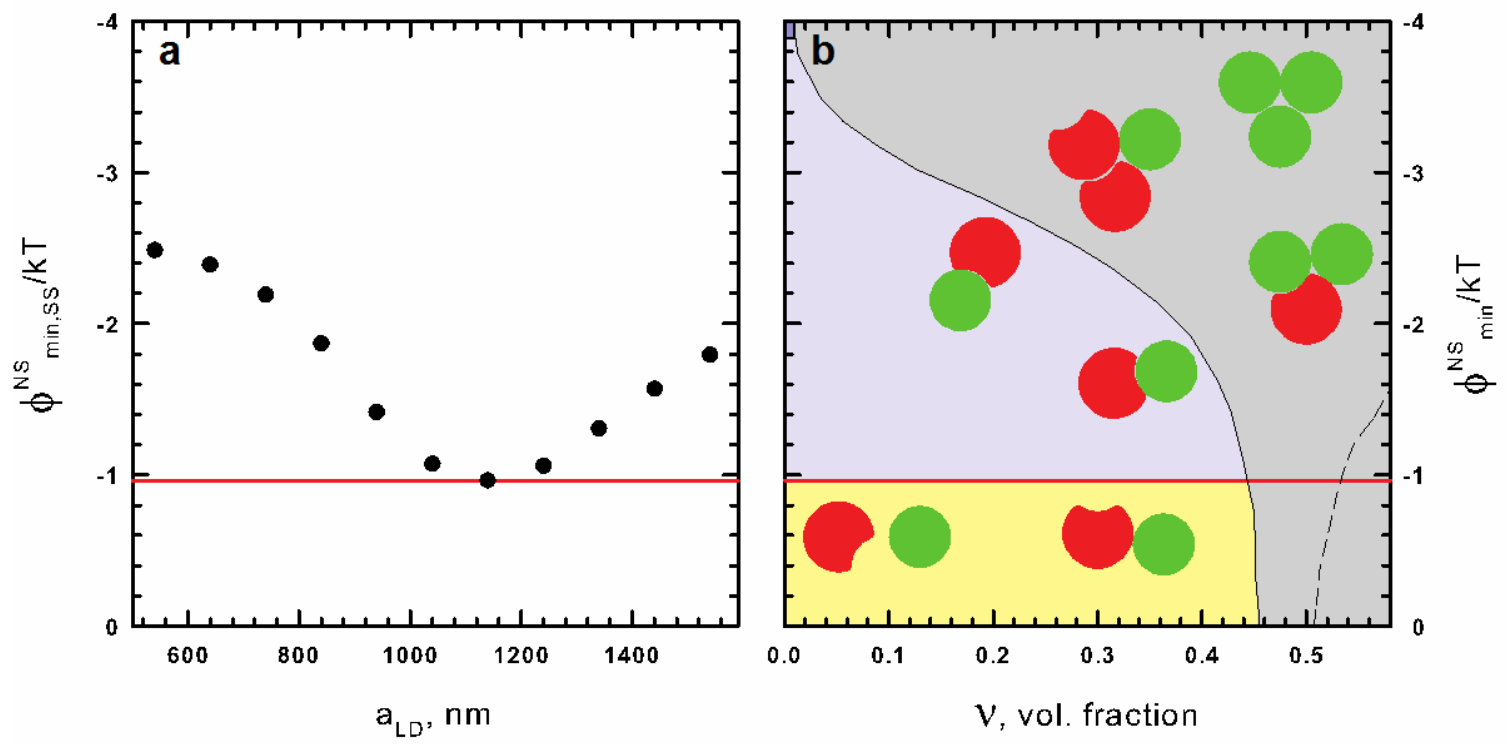

Fig 7. Design chart for dumbbell assembly. (a) threshold minimum non-specific potential energy, to favor specific binding $\left(\Delta F_{\mathrm{NS}-\mathrm{S}}<0\right), \phi \square_{\text {min,SS}}$, as a function of lock dimple radius, $a_{\mathrm{LD}}$. (b) phase diagram for hard spheres with depletion potential using the B2 device[34] in non-specific potential well depth vs. packing fraction $\left(\phi \square_{\min }-v\right)$ space. The solid black lines denotes the freezing line, while the dashed black lines denotes the melting line for fluid-solid coexistence[35]. The shaded regions denote different regimes of dumbbell assembly: aggregation region, grey; unstable specific binding region, yellow; operating window, i.e. stable specific binding region, blue. The red lines are tie lines between panels (a) and (b) denoting the minimum non-specific $\phi{ }_{\text {min }}$ to favor specific binding for $a_{\mathrm{LD}}=1140 \mathrm{~nm}$.

Very high interaction strength combined with large particle concentrations can lead to aggregation and possible frustration of the formation of specific bonds. The aggregation line can be estimated as the freezing line (i.e. the solid black line) in the phase diagram. In Figure $7 \mathrm{~b}$ we show the phase space for lock and key particles in terms of non-specific interaction strength, $\phi^{\mathrm{NS}}{ }_{\text {min }}$, versus total colloid volume fraction, $v$. In Fig $7 \mathrm{~b}$ we show freezing and melting lines for an equivalent colloid system with depletion interactions[34,35], for a system with a particle size $a=0.5\left(a_{\mathrm{L}}+a_{\mathrm{K}}\right)$. For concentrations above the freezing line, multi-body interactions will dominate and formation of specific pairs can be hindered. With aggregation and unstable specific bonds identified we can show where the "operating window" for dumbbell formation is, as shown in shaded blue region in Fig 7b. The aggregation zone is shown in shaded grey, while the weak specific bond region is shown in yellow shaded region.

In addition to identifying the operating window, kinetic information can also guide the region in phase space where binding can occur faster. Firstly, free to specific and to non-specific kinetics are maximized by increasing the total colloidal volume fraction. While kinetic rate constants do not change with colloid volume fraction, as specified in Eqs (18)-(21), the total rate 
does. Secondly, as shown in Fig 5d, non-specific to free kinetics decrease with increasing nonspecific interaction strength. Given the low success probabilities of non-specific to specific events, increasing the rate of non-specific to free events increases the total yield by enabling the free-specific kinetics. Thus, low $\phi^{\mathrm{NS}}{ }_{\min }$ (but above $\phi^{\mathrm{NS}}{ }_{\text {min,SS }}$ ) and high $v$ (but below the freezing line) ensure fast formation of specific pairs.

\section{Experimental limitations and their impact on model analysis and predictions}

Motivated by the discrepancy between predicted escape (S-NS/F) kinetic rate constants between experimental measurements and our model, in this section we look into limitations present in experiments and their effects on model predictions. Specifically we study: (1) spatial resolution issues, related to changes in kinetic rate coefficients due to uncertainty in particleparticle separation; (2) temporal resolution issues, related to long frame-to-frame time in experiments and its effect on capturing possibly fast processes; (3) polydisperisty of geometric parameters, specifically lock particle dimple and key particle radius, related to shape complementarity effects studied in a previous section in this paper; and (4) sedimentation of particles, related to possible misidentification of non-specific pairs as specific pairs. Other experimental limitations that proved to have minor effects include changes in lock aperture angle, $\alpha$, changes in rate constants due to quasi-two-dimensional confinement, "depletion barrier" effects[21,36], and shift in hydrodynamic interactions due to adsorbed steric polymer brush layers on particles[37, 38]. These additional, albeit not significant, effects are explained in detail in the Supplementary Material.

\section{Limited spatial resolution effect on NS to F and NS to S kinetics}

Figure 8 shows the kinetic properties of non-specific to free and specific events for a lock aperture angle $\alpha=30^{\circ}$, instantaneous sampling time $\left(\tau_{\mathrm{D}} \rightarrow 0\right)$, non-specific potential energy minimum $\phi^{\mathrm{NS}}{ }_{\min }=2 k T$, and escape distance varying from $h_{\mathrm{a}}=300 \mathrm{~nm}\left(0.25 a_{\mathrm{L}}\right)$ to $500 \mathrm{~nm}\left(0.42 a_{\mathrm{L}}\right)$. Note that the increased escape distance does not imply any change in the range of the attractive potential between the particles, but only the distance within which the particle pair is considered to be non-specifically bound. This distance is much larger than the range of the attractive potential because it is approximately the resolution of the microscopic method used to resolve and discriminate between non-specific and free particle pairs. Fig 8a shows non-specific to free first passage time distributions, $p_{\mathrm{NS}-\mathrm{F}}(t)$, while Fig $8 \mathrm{~b}$ shows non-specific to specific first passage time distributions, $p_{\mathrm{NS}-\mathrm{S}}(t)$. The success probability, as specified in the theory section, is reported in Fig $8 \mathrm{c}$ as a function of $h_{\mathrm{a}}$. The kinetic rate constants for non-specific to free and non-specific to specific transitions, as functions of $h_{\mathrm{a}}$, are shown in Fig $8 \mathrm{~d}$.

The change in escape distance has a very modest effect, as seen in the small change in first passage time distribution changes seen in Figs $8 \mathrm{a}$ and $8 \mathrm{~b}$. It can be seen that with increasing $h_{\mathrm{a}}$ both NS-S and NS-F kinetics become slower, in a similar fashion as increasing potential energy minimum $\phi^{\mathrm{NS}}{ }_{\min }$. Slower transition times are expected with larger $h_{\mathrm{a}}$ values, as it becomes more difficult for NS pairs to become F, which, as discussed before, control the competing process kinetics. As NS to F kinetics become slower, NS pairs are seen to have a higher chance to become $\mathrm{S}$, as observed in increasing success probabilities shown in Fig 8c. The net effects on kinetic rate constants is seen in Fig $8 \mathrm{~d}$ as a net slow-down with increasing $h_{\mathrm{a}}$, but in a more subtle fashion than the change seen as a function of $\phi^{\mathrm{NS}}{ }_{\min }$, seen in Fig $5 \mathrm{~d}$. Given the small changes in both kinetic rate constants and success probabilities due to significant changes in escape distance, $h_{\mathrm{a}}$, we can be confident in kinetic estimations in the light of spatial resolution 
typical for microscopy experiments.
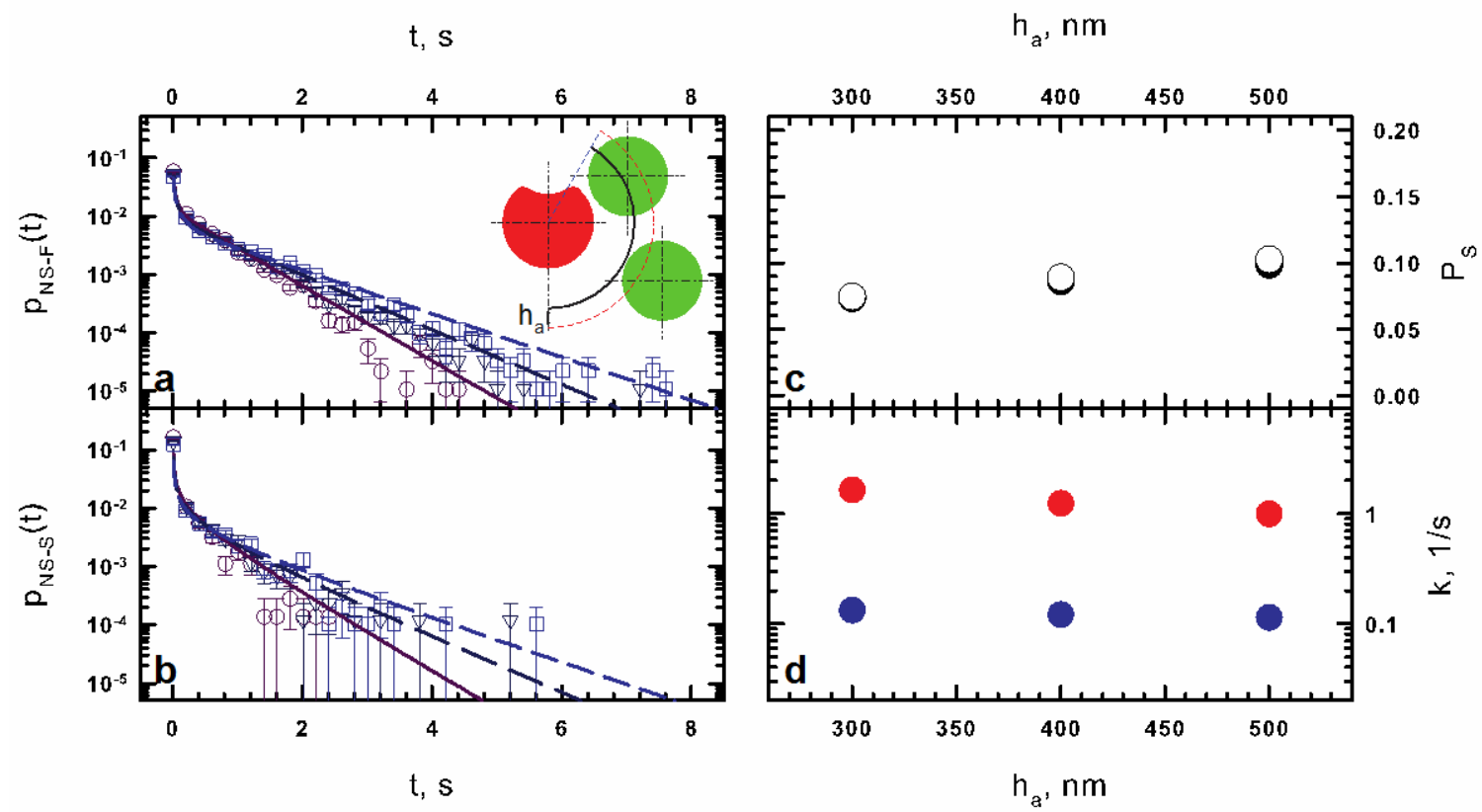

Fig 8. Effect of escape distance, $h_{\mathrm{a}}$, on predicted dynamics. (a) NS to F first passage time distribution, $p_{\mathrm{NS}-\mathrm{F}}$, lines denote diffusion-migration model results, symbols denote simulation results for $\alpha=30^{\circ}, \phi^{\square}{ }_{\text {min }}=2 k T$, $h_{\mathrm{a}}=300 \mathrm{~nm}$ (purple, circles), $h_{\mathrm{a}}=400 \mathrm{~nm}$ (dark blue, triangles), and $h_{\mathrm{a}}=500 \mathrm{~nm}$ (blue, squares). (b) NS to $\mathrm{S}$ first passage time distribution, $p_{\mathrm{NS}-\mathrm{s}}$, for the same cases shown in panel (a). (c) success probabilities, $P_{\mathrm{S}}$, as function of $\alpha$ for the conditions explored in panels (a) and (b) for simulations (white data) and model (black data). (d) kinetic rate constants, $k_{\mathrm{NS}-\mathrm{F}}$ (red) and $k_{\mathrm{NS}-\mathrm{S}}$ (blue), as function of $\alpha$ for the conditions explored in panels (a) and (b).

\section{Limited temporal resolution effect on NS to F and NS to S kinetics}

Limited temporal resolution refers to long time between consecutive frames compared with typical event life times in microscopy experiments performed to analyze rapid dynamic processes[14]. The physical picture of limited temporal resolution is that of a NS-F transition followed by a F-NS transition, both occurring at times shorter than the discreet sampling time, $\tau_{\mathrm{D}}$; it effectively means that a NS-F transition was not accounted for in the experiment and leads to longer measured transition times and distorted kinetics. Figures $9 \mathrm{a}$ and $9 \mathrm{~b}$ show first passage time distributions for non-specific to free, in Fig 9a, and non-specific to specific, in Fig 9b, events for cases with non-specific potential energy minimum $\phi^{\mathrm{NS}}{ }_{\min }=2 \mathrm{kT}$, escape distance $h_{\mathrm{a}}=300 \mathrm{~nm}$, lock aperture angle $\alpha=30^{\circ}$ and discreet sampling time varying from $\tau_{\mathrm{D}}=1.0 \mathrm{~ms}$ (essentially instantaneous sampling) to $\tau_{\mathrm{D}}=1000.0 \mathrm{~ms}$ (which is similar to experimental sampling times[14]). Fig $9 \mathrm{c}$ shows success probabilities as function of $\tau_{\mathrm{D}}$ for the cases explored in Figs $9 \mathrm{a}$ and $9 \mathrm{~b}$. Kinetic rate constants, $k_{\mathrm{NS}-\mathrm{F}}$ and $k_{\mathrm{NS}-\mathrm{S}}$, are shown in Fig $9 \mathrm{~d}$ as functions of $\tau_{\mathrm{D}}$ for the same cases explored in Figs 9a and 9b.

The effect of increasing the discreet sampling time is similar to that of increasing the non-specific potential energy minimum $\phi^{\mathrm{NS}}{ }_{\text {min }}$ or the escape distance $h_{\mathrm{a}}$, that is firstly NS-F and NS-S event life times become larger, as seen in Figs $9 a$ and $9 b$, and secondly the success probability increases as $\tau_{\mathrm{D}}$ increases. The slow-down in transition times is related to the 
possibility that a NS pair transitions fast to a F configuration, but the lock-key pair can return to a NS configuration before a time $\tau_{\mathrm{D}}$ has elapsed, making it an effective longer-lasting NS to F (or S) event. As NS-F kinetics slow down, as seen in Fig 9a, NS-S kinetics also slow down, as seen in Fig 9b, as NS-F kinetics still dominate the competing processes. These longer-lasting NS pairs also imply that the $\mathrm{S}$ state can be reached with a higher probability, increasing the success probability as shown in Fig 9c. The net effect in apparent kinetic rate constants is a decrease in the kinetic rate constant $k_{\mathrm{NS}-\mathrm{F}}$, while $k_{\mathrm{NS}-\mathrm{S}}$ remains fairly constant, as seen in Fig $9 \mathrm{~d}$. Temporal resolution issues, if not accounted for in the analysis of transition kinetics, lead to significant distortions of kinetic information and need to be accounted for.
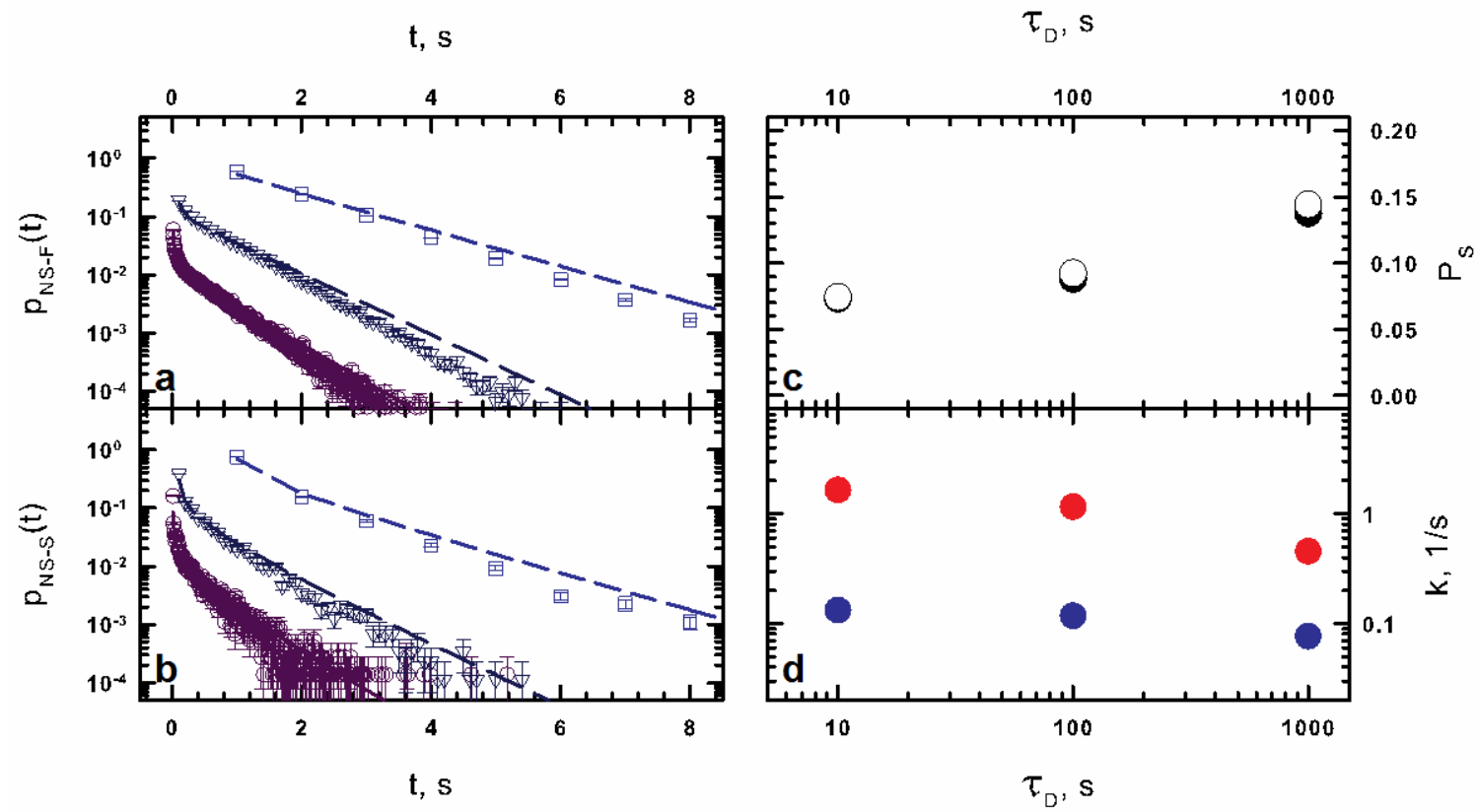

Fig 9. Effect of discrete sampling time, $\tau_{\mathrm{D}}$, on predicted dynamics. (a) NS to $\mathrm{F}$ first passage time distribution, $p_{\mathrm{NS}-\mathrm{F}}$, lines denote diffusion-migration model results, symbols denote simulation results for $\alpha=30^{\circ}, \phi{ }_{\text {min }}=2 k T, h_{\mathrm{a}}=300 \mathrm{~nm}, \tau_{\mathrm{D}}=10 \mathrm{~ms}$ (purple, circles), $\tau_{\mathrm{D}}=100 \mathrm{~ms}$ (dark blue, triangles), and $\tau_{\mathrm{D}}=1000 \mathrm{~ms}$ (blue, squares). (b) NS to S first passage time distribution, $p_{\mathrm{NS}-\mathrm{S}}$, for the same cases shown in panel (a). (c) success probabilities, $P_{\mathrm{S}}$, as function of $\alpha$ for the conditions explored in panels (a) and (b) for simulations (white data) and model (black data). (d) kinetic rate constants, $k_{\mathrm{NS}-\mathrm{F}}$ (red) and $k_{\mathrm{NS}-\mathrm{S}}$ (blue), as function of $\alpha$ for the conditions explored in panels (a) and (b).

Experimental variability in lock dimple geometry effect on apparent escape kinetics and specific pair stability

As shown previously in this paper, the estimation of escape kinetic rate constants, i.e. $k_{\mathrm{S}-\mathrm{F}}$ and $k_{\mathrm{S}-\mathrm{NS}}$, depends on the interparticle potential between lock and key, which in turn depends on the geometric details of both lock and key particles. Variability on the size of particles, and geometric parameters can lead to kinetic behavior drastically different from nominal or average geometry estimations. As an example of the kind of variability observed for lock particles belonging to the same batch, Figure 10 shows two lock particle micrograph examples, where the outer lock radius, $a_{\mathrm{L}}$, and other geometric parameters, $a_{\mathrm{LL}}$ in particular, are seen to vary from particle to particle. The particle batch in question corresponds to experiments documented in a 
previous publication[14].

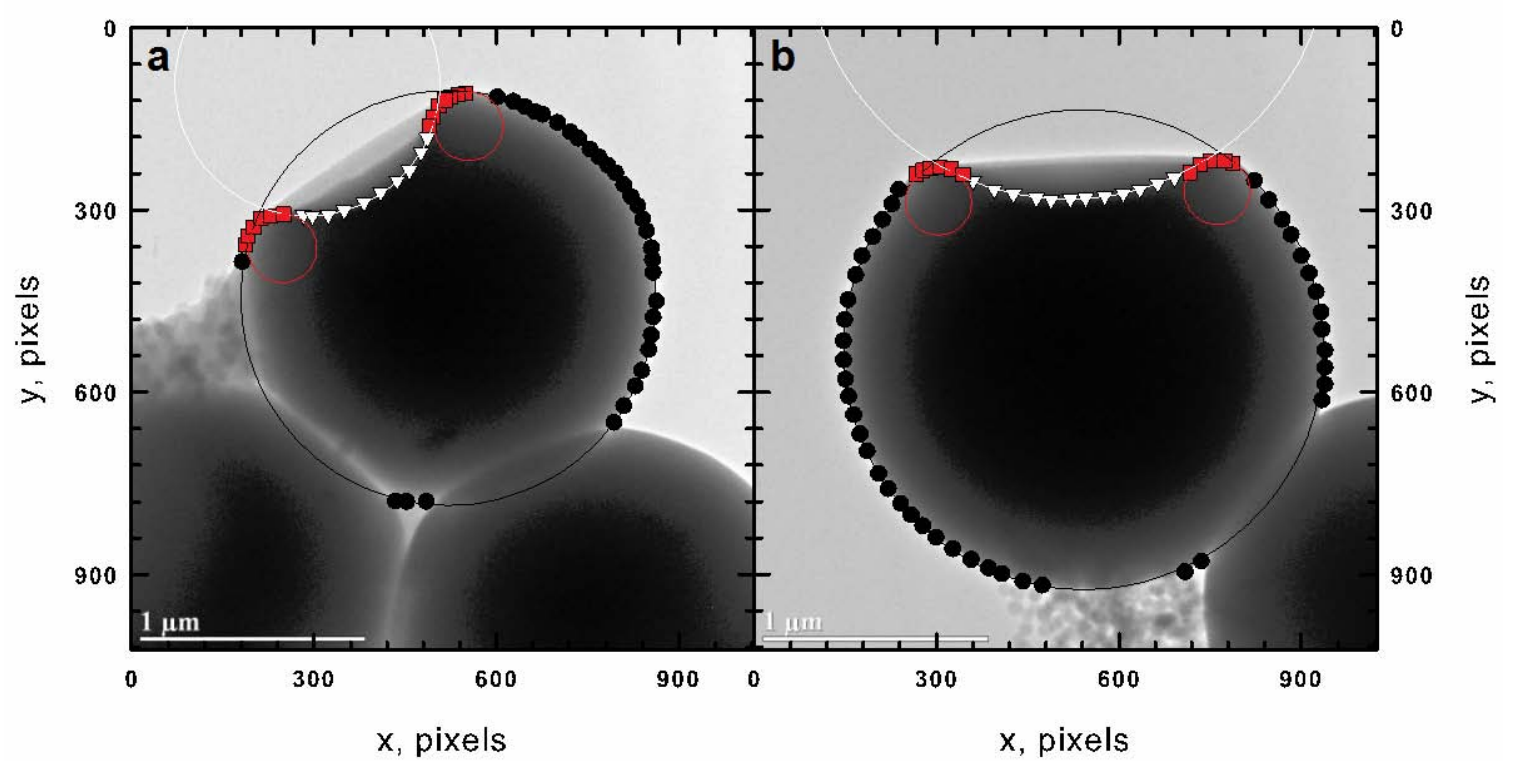

Fig 10. TEM micrographs of two different lock particles showing the similar curvature fitting as that shown in Fig 4a, showcasing the variability in lock particle geometry.

Table 2 shows mean, standard deviation, and polydispersity (defined as the ratio of standard deviation to mean value) of different parameters for $n=4$ lock particles. These statistics are meant to be exemplary situations where the largest variability in parameters can be observed. Of the different parameters shown in Table 2, the lock dimple radius, $a_{\mathrm{LD}}$, shows the largest variability ( $>20 \%$ polydispersity), which warrants further discussion.

\begin{tabular}{|c|c|c|c|}
\hline Parameter & Mean & Standard deviation & Polydispersity \\
\hline$a_{\mathrm{L}}(\mathrm{nm})^{\mathrm{a}}$ & 1200 & 82 & 0.069 \\
\hline$a_{\mathrm{LD}}(\mathrm{nm})^{\mathrm{b}}$ & 1044 & 287 & 0.275 \\
\hline$a_{\mathrm{LL}}(\mathrm{nm})^{\mathrm{c}}$ & 173 & 21 & 0.122 \\
\hline$z_{\mathrm{D}}(\mathrm{nm})^{\mathrm{d}}$ & 744 & 63 & 0.063 \\
\hline$\alpha(\text { degrees })^{\mathrm{e}}$ & 41 & 1.9 & 0.048 \\
\hline
\end{tabular}

Table 2. Lock geometric parameter variability. ${ }^{a}$ Lock particle radius. ${ }^{b}$ Lock particle dimple radius. ${ }^{\mathrm{c}}$ Lock particle lip radius. ${ }^{\mathrm{d}}$ Lock particle center-to-lip basin distance. ${ }^{\mathrm{e}}$ Lock particle aperture angle (defined as tan ${ }^{-}$ $\left.{ }^{1}\left(x_{\mathrm{L}} / z_{\mathrm{L}}\right)\right)$.

The effect of a wide lock dimple size distribution can be quantified by estimating an

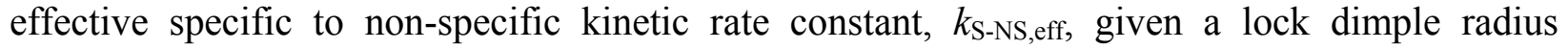
distribution, $p\left(a_{\mathrm{LD}}\right)$ as

$$
k_{S-N S, e f f}=\int d a_{L D} k_{S-N S}\left(a_{L D}\right) p\left(a_{L D}\right)
$$


where a lock dimple radius distribution follows a Gaussian distribution with specified mean and standard deviation. We compute $k_{\mathrm{S}-\mathrm{NS} \text {,eff }}$ for a mean lock dimple radius $a_{\mathrm{LD} \text {,avg }}=1050 \mathrm{~nm}$, and a polydispersity of $20 \%$ and show it next to individual monodisperse estimations in Fig $6 \mathrm{~b}$. We see an effective increase of escape kinetic rate constant for moderate values of the interparticle potential, relative to that for a monodisperse dimple radius with a mean size, $a_{\mathrm{LD}, \mathrm{avg}}=1050 \mathrm{~nm}$. From this effective kinetic rate constant we can the estimate an effective free energy difference as

$$
\Delta F_{N S-S, e f f}=-k T \ln \left(\frac{k_{N S-S}}{k_{S-N S, e f f}}\right),
$$

and show it alongside monodisperse estimation in Fig 6a. The effective free energy difference is shown to have a net increase, as compared with mean size $\left(a_{\mathrm{LD} \text {,avg }}=1050 \mathrm{~nm}\right)$ estimations. The threshold interaction strength, $\phi^{\mathrm{NS}}{ }_{\text {min,SS }}$, is shown to shift to more negative values, as compared with $a_{\mathrm{LD} \text {,avg }}$ estimates. The effect of lock dimple radius brings the estimates of both escape kinetics and apparent free energy differences closer to experimental measurements, but do not explain the high apparent escape rates and small specific to non-specific free energy differences, especially at high non-specific interaction strength.

Sedimentation of lock and key particles effect on misidentification of non-specific and specific pairs

Figure 11a shows the top view of a lock and key pair, where the apparent center-to-center distance, as seen from a two-dimensional image, identifies the particles as a specific pair. A side view of the same particle pair shows, in Fig $11 \mathrm{~b}$, that the pair is actually a non-specific one. Given that a number of non-specific pairs will appear to be false specific pairs, hereafter called false positives, the apparent S-NS kinetic rate constant can include a number of false positives which can have false positive S-NS rates faster than real S-NS transitions. To estimate the rate of false S-NS transitions we use the diffusion-migration model developed for NS-S/F transitions, where the Smoluchowski equation (Eq (22)) is solved on the variable pair $h=r-a_{\mathrm{L}}-a_{\mathrm{K}}, \psi$, where $\psi$ is the angle between the " $z$ " axis and the center-to-center vector between lock and key particles, as shown in Fig 11b. False positive specific pairs are those for which the apparent center-tocenter distance, $r_{\mathrm{app}}=\left(r-a_{\mathrm{L}}-a_{\mathrm{K}}\right) \sin \psi$, is smaller than a distance $r_{\min }=a_{\mathrm{L}}+a_{\mathrm{K}}$. Apparent non-specific pairs are those where $r_{\min }<r_{\text {app }}<h_{\mathrm{a}}+a_{\mathrm{L}}+a_{\mathrm{K}}$. Finally apparent free pairs are those where $r_{\mathrm{app}}>h_{\mathrm{a}}+a_{\mathrm{L}}+a_{\mathrm{K}}$. The potential of mean force required to solve the Smoluchowski equation is the same one stated in Eq (25), with the addition of a gravitational potential for the key, resulting in

$$
W^{\prime}(h, \psi)=\phi^{N S}(h)+G_{K} z+W_{g}(h, \psi),
$$

where $G_{\mathrm{K}}$ refers to the buoyant weight of key particles (having a density of $1.27 \mathrm{~g} / \mathrm{L}[14]$ ) and $z=-$ $r \cos \psi$. The diffusivity tensor, $\mathbf{D}$, is estimated with $\mathrm{Eq}(27)$ and other relevant parameters are summarized in Table 1.

To include the effect of false positives in S-NS kinetic constant estimations we weigh the contribution of real S-NS transitions with different populations of false positive S-NS transitions as 


$$
k_{S-N S, \text { eff }}^{F P}=\left(1-f^{F P}\right) k_{S-N S, e f f}+f^{F P} k_{S-N S}^{F P},
$$

where $k_{\text {S-NS }}^{\mathrm{FP}}$ is the kinetic rate constant for false positive S-NS transitions and $f^{\mathrm{FP}}$ is the fraction of false positive specific pairs in the system. Note that the value of $f^{F P}$ depends on both the likelihood of a NS pair to be perceived as false positive and the total number of NS pairs at some point during an experiment, as shown in the Supplementary Material. The apparent free energy difference between non-specific and specific pairs is given by

$$
\begin{array}{r}
\Delta F_{N S-S, e f f}^{F P}=-k T \ln \left(\frac{k_{N S-S}}{k_{N S-S, e f f}^{F P}}\right) . \\
\phi_{\min ^{N S}} / \mathrm{kT}
\end{array}
$$
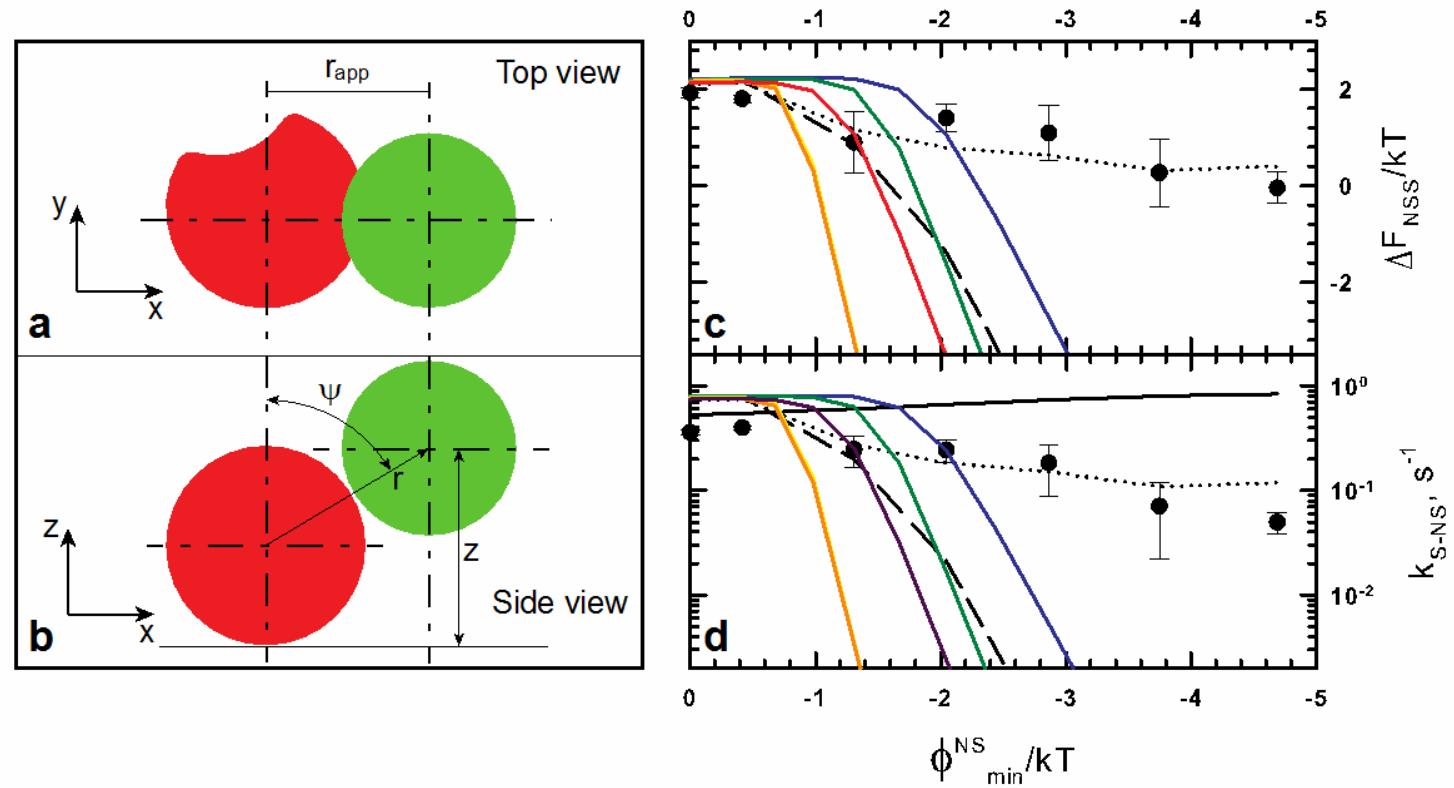

Fig 11. Apparent specific to non-specific kinetic rate constants and its effect on measured escape kinetics and specific pair stability. (a) Schematic of the top view of a non-specific pair that appears to be specific (a false positive) due to the center-to-center distance between lock and key. (b) Schematic of the side view of the same particle pair shown in panel (a). (c) NS to $S$ free energy difference as a function of minimum nonspecific potential energy for different lock dimple radii, $a_{\mathrm{LD}}=840 \mathrm{~nm}$ (blue), $a_{\mathrm{LD}}=940 \mathrm{~nm}$ (green), $a_{\mathrm{LD}}=1040$ (yellow), $a_{\mathrm{LD}}=1140 \mathrm{~nm}$ (orange), $a_{\mathrm{LD}}=1240 \mathrm{~nm}$ (red), apparent free energy difference calculated using $\mathrm{Eq}$ (63) using an $a_{\mathrm{LD}}$ polydispersity of $20 \%$ (dashed black line), apparent free energy difference including false positive specific to non-specific effect using Eq. (66) (dotted black line), as well as experimental data from a previous work[14] (symbols). (d) specific to non-specific kinetic rate constant as a function of minimum non-specific potential energy for the same situations shown in panel (c) in addition to false positive specific to non-specific kinetic rate constant estimations (solid black line).

Figure 11c shows free energy differences between non-specific and specific pairs as estimated using Eq. (63), along with effective free energy differences with $f^{\mathrm{FP}}$ estimated in the Supplementary Material using Eq. (66). Fig 11d shows specific to non-specific kinetic rate constants for the same cases shown in Fig 11c, along with the false positive S-NS kinetic rate constants as functions of non-specific potential energy minimum. Our results show that the false 
positive kinetic rate constant, $k_{\mathrm{S}-\mathrm{NS}}{ }^{\mathrm{FP}}$, has a slightly upward slope when seen as a function of nonspecific potential energy minimum, and its values are comparable to the low interaction strength limit of real S-NS kinetic rate constants. The inclusion of false positives shifts the apparent kinetic rate constant towards values consistent with experimental measurements. The apparent free energy difference shows a similar behavior to kinetic rate constants.

The presence of false positives can lead to a false interpretation of experimental data, as the experimental data shown in Fig 11d, and our previous work[14], suggests poor complementarity between lock pocket and key with free energy between NS and S states being always positive (implying the NS state is always more stable than the S state). The inclusion of false positive kinetics shifts the expected behavior seen in Figs 6 and 11c-d, namely as the nonspecific interaction strength, $\phi_{\mathrm{NS}}{ }^{\mathrm{min}}$, increases the $\mathrm{S}$ state becomes more stable, to the apparently unstable $\mathrm{S}$ states seen in the experimental data. Figure S7 shows evidence of false positive S-NS transitions from the data set used to calculate the symbol data in Figs 11c-d, where it is clear how out-of-plane excursions of key particles can lead to misinterpretation of different types of events.

The physical origin of this shift to unstable $\mathrm{S}$ states can be understood as a small $\left(f^{\mathrm{FP}} \sim 0.2\right.$, as seen in the Supplementary Material) population of false positives undergoes rapid S-NS transitions (shown in the red line in Fig 11d), effectively bringing the overall kinetic rate constant to unnaturally high values. This high apparent kinetic rate constant is then interpreted, using Eq. (66), as S pairs being less stable than what they really are. The real escape kinetics, though, can still be different from our predictions shown in Fig 11d (solid line) because at high $\phi_{\mathrm{NS}}{ }^{\text {min }}$ false positive S-NS transitions dominate the overall response, can mask any errors in the true nature of the transition, and as long as the false positive rate is correctly estimated, a good match to the experimental data in Fig 11d (symbols) would be attained. To get a definite corroboration of the estimates made using Eq. (62) an experimental technique that bypasses the limitations imposed by incorrect identification of specific and non-specific pairs, especially the inability to determine quantitatively the orientation of lock particles, would be required.

\section{Conclusions}

In this work we developed a systematic approach to study the kinetics of lock and key particle assembly as a reaction system between F (free locks and keys), non-specifically (NS) bound pairs and specifically (S) bound pairs. The presented methodology, expanded from our previous study[14], relies on knowledge of interaction potentials between particles, dissipative forces from hydrodynamic interactions and equilibrium thermodynamics. The expanded model includes a theory that allows the estimation of S to NS and S to F transitions, not studied in our previous publication[14], from a combination of known rate constants (F to NS/S and NS to S/F) and free energy differences from arbitrary shape estimations of interaction potentials. Specifically, we (1) benchmarked the expanded model and use it to guide the design of colloidal lock-key binding systems, and (2) visited experimental limitations with the purpose of assessing how spatial and temporal resolution of instruments, geometry size distribution of the particles involved, and the sedimentation of particles in real experimental conditions affect model predictions.

We benchmarked the expanded model by comparing predictions with Stokesian dynamic simulations of NS to S and NS to F transitions and found extremely good agreement between 
model predictions and direct simulation results for all quantities measured. We also studied model predictions to understand the role of shape complementarity in specific pair stability and escape, i.e. S to NS and F, kinetics. We found that optimum shape complementarity occurs when the lock pocket is slightly larger than the key, consistent with an interplay between the strength of the specific bond and the configurational entropy of the key inside the lock. With kinetic and stability model predictions we posed a design scheme to allow the rapid design of colloidal lock and key assemblies in terms of (1) enabling the preferential formation of specific pairs by working at conditions where specific bonds are preferred over non-specific bonds, (2) preventing the formation of many-body aggregates rather than specific pairs, and (3) maximizing specific pair formation rates. The study of experimental non-idealities is motivated by direct comparisons with experimentally determined kinetic rates measured in a previous confocal microcopy study[14] and the effect of these non-idealities on model predictions and interpretation. The main factors affecting model estimations of kinetic rate constants were found to be temporal resolution, particle geometry polydispersity, and out-of-plane excursions of particles that cause misidentification of specific bonds. Additional non-idealities with the potential to bias the analysis of binding kinetics were studied, albeit their effect was found to be minimal.

The kinetic model presented, benchmarked, and experimentally validated here is unique as it allows unprecedented predictive power, compared with previous experimental[13] and theoretical[16] studies, of colloidal lock and key binding systems. Besides benchmarking the model with state of the art simulation techniques and measurements, including experimental nonidealities, we show the design of lock and key binding systems based on the acquired knowledge, which is key to the optimal formation of dumbbells, which themselves are building blocks in the hierarchical assembly of complex crystalline structures. Furthermore, the understanding of colloidal lock and key assembly can be extrapolated to different length and time scales to important phenomena such as enzyme-ligand and antibody-antigen binding. Further work in this area includes the estimation of the hydrodynamic interactions due to the non-spherical shape of the lock and its effects on both binding and escape kinetics, multi-body effects that lead to the formation of higher order aggregates (trimers, tetramers, etc.) and the design of such systems, and the assessment of hierarchical assembly schemes to produce large periodic arrays.

\section{Acknowledgments}

All authors acknowledge support from the US Army Research Office through a MURI grant (Award number W911NF10-1-0518).

\section{References}

[1] S.C. Glotzer, M.J. Solomon, Anisotropy of building blocks and their assembly into complex structures, Nature materials, 6 (2007) 557-562.

[2] A. Cuetos, M. Dijkstra, Kinetic pathways for the isotropic-nematic phase transition in a system of colloidal hard rods: A simulation study, Physical Review Letters, 98 (2007).

[3] S. Sacanna, L. Rossi, A. Wouterse, A.P. Philipse, Observation of a shape-dependent density maximum in random packings and glasses of colloidal silica ellipsoids, Journal of PhysicsCondensed Matter, 19 (2007).

[4] P.F. Damasceno, M. Engel, S.C. Glotzer, Predictive self-assembly of polyhedra into complex structures, Science, 337 (2012) 453-457.

[5] Z.L. Zhang, S.C. Glotzer, Self-assembly of patchy particles, Nano Letters, 4 (2004) 14071413. 
[6] Z. Preisler, T. Vissers, F. Smallenburg, G. Munao, F. Sciortino, Phase Diagram of One-Patch Colloids Forming Tubes and Lamellae, Journal of Physical Chemistry B, 117 (2013) 9540-9547.

[7] Q. Chen, J.K. Whitmer, S. Jiang, S.C. Bae, E. Luijten, S. Granick, Supracolloidal Reaction Kinetics of Janus Spheres, Science, 331 (2011) 199-202.

[8] Q. Chen, S.C. Bae, S. Granick, Directed self-assembly of a colloidal kagome lattice, Nature, 469 (2011) 381-384.

[9] D.J. Beltran-Villegas, B.A. Schultz, N.H. Nguyen, S.C. Glotzer, R.G. Larson, Phase behavior of Janus colloids determined by sedimentation equilibrium, Soft matter, 10 (2014) 4593-4602.

[10] L. Filion, M. Marechal, B. van Oorschot, D. Pelt, F. Smallenburg, M. Dijkstra, Efficient Method for Predicting Crystal Structures at Finite Temperature: Variable Box Shape Simulations (vol 103, 188302, 2009), Physical Review Letters, 103 (2009).

[11] B. Peters, Competing nucleation pathways in a mixture of oppositely charged colloids: Outof-equilibrium nucleation revisited, The Journal of chemical physics, 131 (2009) 244103.

[12] I.C. Jenkins, M.T. Casey, J.T. McGinley, J.C. Crocker, T. Sinno, Hydrodynamics selects the pathway for displacive transformations in DNA-linked colloidal crystallites, Proceedings of the National Academy of Sciences, 111 (2014) 4803-4808.

[13] S. Sacanna, W. Irvine, P.M. Chaikin, D.J. Pine, Lock and key colloids, Nature, 464 (2010) 575-578.

[14] L. Colón-Meléndez, D.J. Beltran-Villegas, G. van Anders, J. Liu, M. Spellings, S. Sacanna, D.J. Pine, S.C. Glotzer, R.G. Larson, M.J. Solomon, Binding kinetics of lock and key colloids, The Journal of chemical physics, 142 (2015) 174909.

[15] S. Sacanna, M. Korpics, K. Rodriguez, L. Colón-Meléndez, S.-H. Kim, D.J. Pine, G.-R. Yi, Shaping colloids for self-assembly, Nature communications, 4 (2013) 1688.

[16] G. Odriozola, M. Lozada-Cassou, Statistical Mechanics Approach to Lock-Key Supramolecular Chemistry Interactions, Physical Review Letters, 110 (2013) 105701.

[17] P.-M. König, R. Roth, S. Dietrich, Lock and key model system, EPL (Europhysics Letters), 84 (2008) 68006.

[18] S. Asakura, F. Oosawa, On interaction between two bodies immersed in a solution of macromolecules, Chemical Physics, DOI (1954) 1255-1256.

[19] W.B. Russel, D.A. Saville, W.R. Schowalter, Colloidal Dispersions, Cambridge University Press 1992.

[20] T.D. Edwards, M.A. Bevan, Polymer mediated depletion attraction and interfacial colloidal phase behavior, Macromolecules, 45 (2011) 585-594.

[21] A. Dinsmore, A. Yodh, D. Pine, Entropic control of particle motion using passive surface microstructures, Nature, 383 (1996) 239-242.

[22] L.R. White, On the Deryaguin approximation for the interaction of macrobodies, Journal of colloid and interface science, 95 (1983) 286-288.

[23] D.J. Jeffrey, Y. Onishi, Calculation of the resistance and mobility functions for two unequal rigid spheres in low-Reynolds-number flow, Journal of Fluid Mechanics, 139 (1984) 261-290.

[24] S. Kim, S.J. Karrila, Microhydrodynamics: Principles and Selected Applications, Dover Publications2013.

[25] H. Risken, The Fokker-Planck Equation: Methods of Solution and Applications, Springer Berlin Heidelberg1996.

[26] G. Hummer, Position-dependent diffusion coefficients and free energies from Bayesian analysis of equilibrium and replica molecular dynamics simulations, New Journal of Physics, 7 (2005) - 
[27] D.J. Beltran-Villegas, R.M. Sehgal, D. Maroudas, D.M. Ford, M.A. Bevan, Colloidal cluster crystallization dynamics, The Journal of chemical physics, 137 (2012) 134901.

[28] J.F. Brady, G. Bossis, Stokesian dynamics, Annual review of fluid mechanics, 20 (1988) 111-157.

[29] J. Liu, R.G. Larson, Brownian dynamics method for simulation of binding kinetics of patterned colloidal spheres with hydrodynamic interactions, The Journal of chemical physics, 138 (2013) 174904.

[30] M. Fixman, Simulation of polymer dynamics. I. General theory, The Journal of Chemical Physics, 69 (1978) 1527-1537.

[31] P. Grassia, E. Hinch, L. Nitsche, Computer simulations of Brownian motion of complex systems, Journal of Fluid Mechanics, 282 (1995) 373-403.

[32] S.G. Anekal, M.A. Bevan, Interpretation of conservative forces from Stokesian dynamic simulations of interfacial and confined colloids, J Chem Phys, 122 (2005) -.

[33] G.M. Whitesides, V.M. Krishnamurthy, Designing ligands to bind proteins, Quarterly reviews of biophysics, 38 (2005) 385-395.

[34] M.A. Bevan, S.N. Petris, D.Y. Chan, Solvent quality dependent continuum van der Waals attraction and phase behavior for colloids bearing nonuniform adsorbed polymer layers, Langmuir, 18 (2002) 7845-7852.

[35] S.M. Ilett, A. Orrock, W. Poon, P. Pusey, Phase behavior of a model colloid-polymer mixture, Physical Review E, 51 (1995) 1344.

[36] Y. Yang, T.D. Edwards, M.A. Bevan, Modeling depletion mediated colloidal assembly on topographical patterns, Journal of colloid and interface science, 449 (2015) 270-278.

[37] A. Potanin, W. Russel, Hydrodynamic interaction of particles with grafted polymer brushes and applications to rheology of colloidal dispersions, Physical Review E, 52 (1995) 730.

[38] D.J. Beltran-Villegas, T.D. Edwards, M.A. Bevan, Self-consistent colloidal energy and diffusivity landscapes in macromolecular solutions, Langmuir, 29 (2013) 12337-12341. 